\title{
ON THE GEOMETRY OF THE SOLUTIONS OF THE COVER PROBLEM
}

\author{
F. PUERTA ${ }^{1 *}, \mathrm{X}$. PUERTA ${ }^{1 \dagger}$, AND I. ZABALLA ${ }^{2 \ddagger}$
}

\begin{abstract}
For a given system $(A, B)$ and a subspace $\mathcal{S}$, the Cover Problem consits of finding all $(A, B)$-invariant subspaces containing $\mathcal{S}$. For controllable systems, the set of these subspaces can be suitably stratified. In this paper, necessary and sufficient conditions are given for the cover problem to have a solution on a given strata. Then the geometry of these solutions is studied. In particular, the set of the solutions is provided with a differentiable structure and a parametrization of all solutions is obtained through a coordinate atlas of the corresponding smooth manifold.
\end{abstract}

Key words. Cover problem, controlled invariant subspace, smooth manifold, coordinate chart, Brunovsky indices.

AMS subject classifications. 93B29,15A03.

1. Introduction. Given a time-invariant linear system

$$
\left\{\begin{array}{l}
\dot{x}(t)=A x(t)+B u(t) \\
y(t)=C x(t)
\end{array}\right.
$$

a subspace $\mathcal{V}$ is said to be $(A, B)$-invariant (or controlled invariant as in[3]) if $A \mathcal{V} \subset \mathcal{V}+\operatorname{Im} B$

For a given subspace $\mathcal{S}$, the cover problem consists of finding all $(A, B)$-invariant subspaces $\mathcal{V}$ such that $\mathcal{S} \subset \mathcal{V}$. A number of important problems are related to the cover problem. For instance, the disturbance decoupling problem calls for a state feedback transformation such that the output of the closed-loop system does not depend on the input disturbance. As shown in [8, Th. 4.2] this is possible if and only if $\operatorname{Im} Q \subset$ $\operatorname{Im}(A, B ; \operatorname{ker} C)$ where $\operatorname{Im}(A, B ; \operatorname{Ker} C)$ is the unique maximal $(A, B)$-invariant subspace contained in $\operatorname{Ker} C$, and $Q$ is the input disturbance matrix. Clearly an explicit solution of the cover problem will provide us with an explicit solution for the disturbance decoupling problem. Some other important problems, like the observer and model-matching problems, are related to the cover problem as well (see [1]). Actually, as said by Antoulas in this paper: "The cover problems provide a unifying framework for the solution of a number of important feedforwaad as well as feedback problems in linear system theory".

Antoulas' paper is one of the main contributions to the solution of the cover problem. Antoulas links together the cover problem and the nice partial realization problem providing, through the last one, a parametrization of the solutions. Our approach is completely different. We tackle this problem from a geometrical point of view. Namely, by duality, the cover problem is equivalent to finding all $\left(\begin{array}{l}A^{t} \\ B^{t}\end{array}\right)$-invariant subspaces (for notational convenience, from now on we will write $\left(B^{t}, A^{t}\right)$ instead of $\left.\left(\begin{array}{l}A^{t} \\ B^{t}\end{array}\right)\right) \mathcal{V}$ such that $\mathcal{V} \subset \mathcal{S}^{\perp}$. (Recall that $\mathcal{V}$ is $(C, A)$-invariant if $\left.A(\mathcal{V} \cap \operatorname{Ker} C) \subset \mathcal{V}\right)$. Thus, if $G r_{d}(V)$ denotes the Grassman manifold of $d$-dimensional subspaces of $V$, the set of solutions of the cover problem is the intersection $G r_{d}\left(\mathcal{S}^{\perp}\right)$ with the set of $\left(B^{t}, A^{t}\right)$-invariant subspaces of dimension $d$, both manifolds of $G r_{d}\left(\mathbb{K}^{n}\right)$, $\mathbb{K}$ being the field of real or complex numbers. Our goal is to compute explicitly this intersection. To this end, we partition the set of $\left(B^{t}, A^{t}\right)$-invariant subspaces of dimension $d$ into smooth strata by fixing the Brunovsky form of the corresponding restriction of $\left(B^{t}, A^{t}\right)$ (see [3]) and we use the parametrization of these strata given in [4] in order to describe the solutions of the cover problem through the solutions of a set of linear algebraic systems.

We will use the following notation. As said, $\mathbb{K}$ is the field of real or complex numbers and $G r_{d}\left(\mathbb{K}^{n}\right)$ denotes the Grassman manifold of $d$-dimensional subspaces of $\mathbb{K}^{n}$. $\mathcal{M}_{m, n}$ is the set of $m \times n$ matrices with elements in $\mathbb{K}, \mathcal{M}_{n}$ is the set of the $n$-square ones and $\mathcal{M}_{m, n}^{*}$ is the subset of the full column rank $m \times n$ matrices. If $X \in \mathcal{M}_{m, n}^{*}$ then $[X]$ denotes the subspace spanned by the columns of $X$. Finally, if $X \in \mathcal{M}_{m, n}$, $X^{*} \in \mathcal{M}_{n, m}$ is its conjugate transpose.

The structure of this paper is as follows. In section 2 we recall the parametrization of the set of $\left(B^{t}, A^{t}\right)$ invariant subspaces given in [6]. In section 3 we describe the solutions of the cover problem in terms of the solution of a set of algebraic linear systems on the parameters of the mentioned parametrization. From this

*Departament de Matemàtica Aplicada I, Universitat Politècnica de Catalunya, Diagonal 647, 08028 Barcelona, Spain. e-mail: puerta@ma1.upc.es. Partially supported by the MCyT, Proyecto de Investigación BFM2001-0081-C03-03

${ }^{\dagger}$ Departament de Matemàtica Aplicada I, Universitat Politècnica de Catalunya, Diagonal 647, 08028 Barcelona, Spain. e-mail: coll@ma1.upc.es. Partially supported by the MCyT, Proyecto de Investigación BFM2001-0081-C03-03

${ }^{\ddagger}$ Departamento de Matemática Aplicada y EIO, Universidad del País Vasco, Apartado 640, 48080 Bilbao, Spain. e-mail: mepzatej@lg.ehu.es. Partially supported by the MCyT, Proyecto de Investigación BFM2001-0081-C03-01 
description we derive explicit necessary and sufficient conditions for the cover problem to have a solution with a given observability indices (Theorem 3.4). In section 4 we prove a simple necessary and sufficient condition in order to ensure that the above conditions are satisfied generically (Theorem 4.9). In section 5 we show that the set of solutions of the cover problem is a stratified smooth manifold with an orbit space structure similar to the set of $\left(B^{t}, A^{t}\right)$-invariant subspaces and we compute its dimension (Theorem 5.1). Finally we parameterize this manifold by means of coordinate charts (Theorem 5.9) and we prove that it is connected.

2. Preliminaries. All along this paper a pair $(A, B)$ will be given and we will consider a fixed basis of $\mathbb{K}^{n}$ : the Brunovsky basis with respect to $\left(\begin{array}{c}A^{t} \\ B^{t}\end{array}\right)$ as given in [6]. Actually this is the basis for which system $(A, B)$ is in the Brunovsky form (dual to the usual one) as given in [2, Lemma 4.4]

On one hand recall that $\mathcal{V}$ is an $(A, B)$-controlled invariant subspace if and only if $\mathcal{V}^{\perp}$ is a $\left(B^{t}, A^{t}\right)$ conditioned invariant subspace. And, on the other hand, notice that $\mathcal{S} \subset \mathcal{V}$ if and only if any vector of $\mathcal{V}^{\perp}$ is orthogonal to any vector of $\mathcal{S}$. This means that if $\mathcal{V}^{\perp}=[X]$ and $\mathcal{S}=[F]$ then $X^{*} F=0$. Thus, we can restate the Cover Problem as follows: Given $\mathcal{S} \in G r_{d}\left(\mathbb{K}^{n}\right)$ and a matrix $F \in \mathcal{M}_{d, n}^{*}$ such that $\mathcal{S}=\left[F^{*}\right]$, find all matrices $X \in \mathcal{M}_{n, \delta}^{*}$ such that $[X]$ is a $\left(B^{t}, A^{t}\right)$-conditioned invariant subspace and $F X=0$.

It turns out that the set of matrices $X \in \mathcal{M}_{n, \delta}^{*}$ whose columns form a basis of some $\left(B^{t}, A^{t}\right)$-invariant subspace has been parameterized in [6]. We will take advantage of this fact to characterize the solutions of the matrix equation $F X=0$ whose columns span a $\left(B^{t}, A^{t}\right)$-invariant subspace.

We recall first the basic results about the differentiable structure of the manifold of $\left(B^{t}, A^{t}\right)$-invariant subspace with specific Brunovsky form for the restriction (see [6] for more details and proofs). Given an observable pair $(C, A) \in \mathcal{M}_{q, n} \times \mathcal{M}_{n}$ with observability indices $\underline{k}=\left(k_{1}, \ldots, k_{r}\right)$ and a set of indices $\underline{h}=\left(h_{1}, \ldots, h_{s}\right)$ we will say that $\underline{h}$ is compatible with $\underline{k}$ if $s \leq r$ and $h_{i} \leq k_{i}$ for $i=1,2, \ldots$.

Let the conjugate partitions of $\underline{k}$ and $\underline{h}$ be $\underline{r}=\left(r_{1}, \ldots, r_{k}\right)$ and $\underline{s}=\left(s_{1}, \ldots, s_{h}\right)$, respectively, with $r=r_{1} \geq \ldots \geq r_{k}>0$ and $s=s_{1} \geq \ldots \geq s_{h}>0$. (Recall that if $\underline{k}=\left(k_{1}, k_{2}, \ldots\right)$ then $\underline{r}=\left(r_{1}, r_{2}, \ldots\right)$ is its conjugate partition if and only if $r_{j}=\#\left\{i: k_{i} \geq j\right\}$, where \# stands for cardinality). From now on we will assume that $s_{i}:=0$ for $i>h$ and $r_{i}:=0$ for $i>k$. If $\left(k_{1}, \ldots, k_{r}\right)$ are the observability indices of $(C, A)$ then $\left(r_{1}, \ldots, r_{k}\right)$ are called the Brunovsky indices of $(C, A)$ (see [4]).

Notice that $k_{i} \geq h_{i}$ for $i=1,2, \ldots, r$ if and only if $r_{i} \geq s_{i}$ for $i=1,2, \ldots, k$. In other words $\underline{h}$ and $\underline{k}$ are compatible if and only if $\underline{s}$ and $\underline{r}$ are compatible. In the sequel we also assume that $\underline{s}$ and $\underline{r}$ are compatible partitions and we denote by $\operatorname{Inv}(\underline{r}, \underline{s})$ the set of $(C, A)$-conditioned invariant subspaces for which the restriction of $(C, A)$ to each one of them has Brunovsky indices $\underline{s}$ (see [5] or [6] for the definition of this restriction).

Definition 2.1. .- (i) Let $\mathcal{M}(\underline{r}, \underline{s})$ denote the set of matrices $X \in \mathcal{M}_{n, d}$ which are partitioned into blocks $X=\left(X_{i j}\right), 1 \leq i \leq k, 1 \leq j \leq h$ in such a way that

(a) $X_{i j} \in \mathcal{M}_{r_{i}, s_{j}}$.

(b) $X_{i j}=0$ if $i<j$.

(c) If $i \geq j, X_{i j}$ can be partitioned into blocks $X_{i j}=\left(Z_{i \alpha}^{i-j+1}\right), 1 \leq \alpha \leq h-j+1$, where

$$
Z_{i \alpha}^{i-j+1}=\left(\begin{array}{c}
Y_{i \alpha}^{i-j+1} \\
0
\end{array}\right)
$$

with $Y_{i \alpha}^{i-j+1}$ having size $r_{h-\alpha+i-j+1} \times\left(s_{h-\alpha+1}-s_{h-\alpha+2}\right)$.

(d) $X_{i+1, j+1}$ is obtained from $X_{i j}$ by removing the last $s_{j}-s_{j+1}$ columns and the last $r_{i}-r_{i+1}$ rows for $1 \leq i \leq k, 1 \leq j \leq h$.

(e) $\operatorname{rank} X_{i i}=s_{i}, 1 \leq i \leq k$.

The block decomposition of $X_{i j}$ in (c) will be called its standard block decomposition.

(ii) We denote $\mathcal{G}(\underline{s}):=\mathcal{M}(\underline{s}, \underline{s})$.

Perhaps a more intuitive description of a matrix $X \in \mathcal{M}(\underline{r}, \underline{s})$ is as follows: $X=\left(X_{i j}\right), 1 \leq i \leq k$, $1 \leq j \leq h$ where:

1. $X_{i j} \in \mathcal{M}_{r_{i}, s_{j}}$.

2. $X_{i j}=0$ if $i<j$.

3. For $\ell=1, \ldots, k, X_{\ell 1}=\left(X_{i j}^{\ell}\right), 1 \leq i \leq k-\ell+1,1 \leq j \leq h, X_{i j}^{\ell}$ of size $\left(r_{k-i+1}-r_{k-i+2}\right) \times\left(s_{h-j+1}-\right.$ $\left.s_{h-j+2}\right)$, and $X_{i j}^{\ell}=0$ for $i>k-h-\ell+j+1$. That is to say, if we divide $X_{\ell, 1}$ into $(k-\ell+1) \times h$ blocks of size $\left(r_{k-i+1}-r_{k-i+2}\right) \times\left(s_{h-j+1}-s_{h-j+2}\right), 1 \leq i \leq k-\ell+1,1 \leq j \leq h$, then $X_{\ell, 1}$ is block upper triangular. 
4. $X_{i j}$ is obtained from $X_{i-1, j-1}$ by deleting the last row and column blocks.

EXAMPLE 2.2. .- If $k=4$ and $h=3$ a matrix $X \in \mathcal{M}(\underline{r}, \underline{s})$ would have the following general form

$\left.\begin{array}{l|ccc|cc|c}r_{4} & X_{31}^{1} & X_{12}^{1} & X_{13}^{1} & & & \\ r_{3}-r_{4} & x_{21}^{1} & X_{22}^{1} & X_{23}^{1} & & & \\ r_{1}-r_{2} & 0 & X_{32}^{1} & X_{33}^{1} & \mathbf{0} & & \mathbf{0} \\ r_{4} & 0 & 0 & X_{43}^{1} & & & \\ r_{3}-r_{4} & r_{2}-r_{3} \\ r_{4} & X_{11}^{2} & X_{12}^{2} & X_{13}^{2} & X_{11}^{1} & X_{12}^{1} & \\ r_{3}-r_{4} & X_{22}^{2} & X_{23}^{2} & X_{21}^{1} & X_{22}^{1} & \mathbf{0} \\ r_{4} & 0 & X_{33}^{2} & 0 & X_{32}^{1} & \\ \hline 0 & X_{12}^{3} & X_{13}^{3} & X_{11}^{2} & X_{12}^{2} & X_{11}^{1} \\ 0 & 0 & X_{23}^{3} & 0 & X_{22}^{2} & X_{21}^{1} \\ \hline 0 & 0 & X_{13}^{4} & 0 & X_{12}^{3} & X_{11}^{2}\end{array}\right)$

And, in this example, a typical element of $\mathcal{G}(\underline{s})$ would be:

$$
\left.\begin{array}{l|ccc|cc|c}
s_{3} & T_{11}^{1} & T_{12}^{1} & T_{13}^{1} & & & \\
s_{2}-s_{3} & T_{1}^{1}-s_{2} & T_{23}^{1} & \mathbf{0} & \mathbf{0} \\
s_{3} & T_{2}-s_{3} \\
s_{3} & 0 & T_{33}^{1} & & & \\
\hline 0 & T_{12}^{2} & T_{13}^{2} & T_{11}^{1} & T_{12}^{1} & \\
0 & 0 & T_{23}^{2} & 0 & T_{22}^{1} & \mathbf{0} \\
\hline 0 & 0 & T_{13}^{3} & 0 & T_{12}^{2} & T_{11}^{1}
\end{array}\right)
$$

Notice that if $r_{i}=r_{i+1}$ or $s_{i}=s_{i+1}$ then some blocks $X_{i j}^{\ell}$ vanish. For example if $\underline{k}=(4,3,2,1,1)$ and $\underline{h}=(3,2,2)$, then $k=4, h=3, \underline{r}=(5,3,2,1)$ and $\underline{s}=(3,3,1)$. In this case, a matrix in $\mathcal{M}(\underline{r}, \underline{s})$ has the following form for any choice of the nonzero parameters:

$$
\left(\begin{array}{ccc|ccc|c}
x_{1} & y_{1} & y_{2} & & & & \\
x_{2} & y_{3} & y_{4} & & & & \mathbf{0} \\
0 & y_{5} & y_{6} & & \mathbf{O} & & \\
0 & 0 & 0 & & & & \\
0 & 0 & 0 & & & & \\
\hline z_{1} & t_{1} & t_{2} & x_{1} & y_{1} & y_{2} & \\
0 & t_{3} & t_{4} & x_{2} & y_{3} & y_{4} & \mathbf{0} \\
0 & 0 & 0 & 0 & y_{5} & y_{6} & \\
\hline 0 & u_{1} & u_{2} & z_{1} & t_{1} & t_{2} & x_{1} \\
0 & 0 & 0 & 0 & t_{3} & t_{4} & x_{2} \\
\hline 0 & 0 & 0 & 0 & u_{1} & u_{2} & z_{1}
\end{array}\right) .
$$

and so $X_{i 3}^{\ell}$ vanishes because $s_{1}=s_{2}=3$.

Notice also that $\operatorname{rank} X_{i i}=s_{i}$ if and only if $\operatorname{rank} X_{11}=s_{1}$.

THEOREM 2.3. .- With the notation of Definition 2.1:

1. $\mathcal{G}(\underline{s})$ is a subgroup of $\mathrm{Gl}\left(\mathbb{K}^{d}\right)$.

2. $\mathcal{G}(\underline{s})$ acts freely on $\mathcal{M}(\underline{r}, \underline{s})$ on the right by matrix multiplication.

3. If $X \in \mathcal{M}(\underline{r}, \underline{s}), T \in \mathrm{Gl}\left(\mathbb{K}^{d}\right)$ and $X T \in \mathcal{M}(\underline{r}, \underline{s})$, then $T \in \mathcal{G}(\underline{s})$.

4. The orbit space $\mathcal{M}(\underline{r}, \underline{s}) / \mathcal{G}(\underline{s})$ has a differentiable structure such that the natural projection $\mathcal{M}(\underline{r}, \underline{s})$ $\longrightarrow \mathcal{M}(\underline{r} ; \underline{s}) / \mathcal{G}(\underline{s})$ is a submersion.

There is a natural bijection between $\operatorname{Inv}(\underline{r}, \underline{s})$ and $\mathcal{M}(\underline{r}, \underline{s}) / \mathcal{G}(\underline{s})$ given by

$$
\begin{array}{ccc}
\mathcal{M}(\underline{r}, \underline{s}) / \mathcal{G}(\underline{s}) & \longrightarrow & \operatorname{Inv}(\underline{r}, \underline{s}) \\
\{X P \mid X \in \mathcal{M}(\underline{r}, \underline{s}), P \in \mathcal{G}(\underline{s})\} & \rightsquigarrow & {[X]}
\end{array}
$$

(In other words every $(C, A)$-conditioned invariant subspace with $\underline{s}$ as Brunovsky indices for the restriction is spanned by the columns of a matrix in $\mathcal{M}(\underline{r}, \underline{s}))$. We consider in $\operatorname{Inv}(\underline{r} ; \underline{s})$ the differentiable structure defined through this bijection. 
Corollary 2.4. .- If for $j=1, \ldots, h, m_{j}=s_{j}-s_{j+1}$ then

$$
\operatorname{dim} \operatorname{Inv}(\underline{r}, \underline{s})=\sum_{i=1}^{h} \sum_{j=1}^{k} m_{i}\left(r_{i+j-1}-s_{i+j-1}\right)
$$

Definition 2.5. .- A matrix $Y \in \mathcal{M}(\underline{r}, \underline{s})$ is said to be in reduced form if there exists a set of pairwise different positive integers $\underline{n}=\left\{n_{i j}, 1 \leq i \leq h, 1 \leq j \leq m_{h-i+1}\right\}$ such that for $i=1,2, \ldots, h$

$$
1 \leq n_{i 1}<n_{i 2}<\ldots<n_{i m_{h-i+1}} \leq r_{h-i+1}
$$

and $Y$ can be partitioned into blocks $Y=\left(Y_{i j}\right), 1 \leq i \leq k, 1 \leq j \leq h$, satisfying the following conditions

1. $Y_{i j} \in \mathcal{M}_{r_{i}, s_{j}}$.

2. $Y_{i j}=0$ if $i<j$.

3. For $i=1,2, \ldots, h, Y_{i i}$ can be partitioned into blocks $Y_{i i}=\left(L_{i \beta}^{1}\right), 1 \leq \beta \leq h-i+1$ in such a way that for $\beta=1,2, \ldots, h-i+1, L_{i \beta}^{1} \in \mathcal{M}_{r_{i}, m_{h-\beta+1}}$ is a matrix whose last $r_{i}-r_{h-\beta+1}$ rows are zero, the rows $n_{i j}, 1 \leq i \leq \beta-1,1 \leq j \leq m_{h-i+1}$ are also zero, and the rows $n_{\beta 1}, n_{\beta 2}, \ldots, n_{\beta m_{h-\beta+1}}$ are unit vectors $e_{1}^{\beta}, e_{2}^{\beta}, \ldots, e_{m_{h-\beta+1}}^{\beta}$ :

$$
e_{j}^{\beta}=\left(\begin{array}{lll}
0 \ldots 0 & \stackrel{(j}{1} & 0 \ldots 0
\end{array}\right) \in \mathbb{K}^{m_{h-\beta+1}} .
$$

4. For $i>j, Y_{i j}$ can be partitioned into blocks $Y_{i j}=\left(L_{i \beta}^{i-j+1}\right), 1 \leq \beta \leq h-j+1$ in such a way that $L_{i \beta}^{i-j+1} \in \mathcal{M}_{r_{i}, m_{h-\beta+1}}$ is a matrix whose last $r_{i}-r_{h-\beta+i-j+1}$ rows are zero and for $\beta \geq i-j+1$, the rows $n_{p q}, 1 \leq p \leq \beta-i+j, 1 \leq q \leq m_{h-p+1}$ are also zero. (Note that the entries of $Y_{i j}$ are prescribed by the entries of $\left.Y_{i-j+1,1}\right)$.

5. The number of parameters of $Y$ coincide with the dimension of $\operatorname{Inv}(\underline{r}, \underline{s})$.

Proposition 2.6. -- For every $X \in \mathcal{M}(\underline{r}, \underline{s})$ there exists a matrix $P \in \mathcal{G}(\underline{s})$ such that $X P$ is a matrix in reduced form. Then, the set of integres $\underline{n}$ is called an admissible set of indices for $X$ and $X P$ a reduced form of $X$.

This admissible set of indices for $X$ depends only on the block $X_{11}$ and completely determines the structure of the reduced form $Y$. However, they are not uniquely determined by $X_{11}$; that is to say, several sets may be admissible for the same matrix. In fact, $1 \leq n_{11}<n_{12}<\cdots<n_{1 s_{h}} \leq r_{h}$ are $s_{h}$ linearly independent rows of the matrix

$$
\left(\begin{array}{c}
X_{11}^{1} \\
X_{21}^{1} \\
\vdots \\
X_{k-h+1,1}^{1}
\end{array}\right)
$$

$1 \leq n_{11}<n_{12}<\cdots<n_{1 s_{h}} \leq r_{h}, 1 \leq n_{21}<n_{22}<\cdots<n_{2 s_{h-1}-s_{h}} \leq r_{h-1}$ are $s_{h-1}$ linearly independent rows of

$$
\left(\begin{array}{cc}
X_{11}^{1} & X_{12}^{1} \\
X_{21}^{1} & X_{22}^{1} \\
\vdots & \vdots \\
X_{k-h+1,1}^{1} & X_{k-h+1,2}^{1} \\
0 & X_{k-h+2,2}^{1}
\end{array}\right),
$$

and so on. Of course, there may be several different forms of choosing these linear independent rows. 
EXAMPLE 2.7. .- For the matrix in example 2.2 if $x_{1} \neq 0$, a reduced form of $X$ is

$$
\left(\begin{array}{ccc|ccc|c}
1 & 0 & 0 & & & \\
x & 1 & 0 & & & \\
0 & 0 & 1 & & \mathbf{0} & \mathbf{0} \\
0 & 0 & 0 & & & & \\
0 & 0 & 0 & & & & \\
\hline y & 0 & 0 & 1 & 0 & 0 & \mathbf{0} \\
0 & z & t & x & 1 & 0 & \mathbf{0} \\
0 & 0 & 0 & 0 & 0 & 1 & \\
\hline 0 & u & v & y & 0 & 0 & 1 \\
0 & 0 & 0 & 0 & z & t & x \\
\hline 0 & 0 & 0 & 0 & u & v & y
\end{array}\right)
$$

where $n_{11}=1, n_{21}=2$ and $n_{22}=3$. And if $x_{2} \neq 0$, another reduced form of $X$ is

$$
\left(\begin{array}{ccc|ccc|c}
x & 1 & 0 & & & \\
1 & 0 & 0 & & & \\
0 & 0 & 1 & & \mathbf{O} & \mathbf{0} \\
0 & 0 & 0 & & & & \\
0 & 0 & 0 & & & & \\
\hline y & z & t & x & 1 & 0 & \mathbf{0} \\
0 & 0 & 0 & 1 & 0 & 0 & \mathbf{O} \\
0 & 0 & 0 & 0 & 0 & 1 & \\
\hline 0 & u & v & y & z & t & x \\
0 & 0 & 0 & 0 & 0 & 0 & 1 \\
\hline 0 & 0 & 0 & 0 & u & v & y
\end{array}\right)
$$

where $n_{11}=2, n_{21}=1, n_{22}=3$. Actually, these are the only two possible cases.

Proposition 2.8. .- (i) Let $X \in \mathcal{M}(\underline{r}, \underline{s})$ and $Q \in \mathcal{G}(\underline{s})$. If $\underline{n}$ is an admissible set of indices for $X$, it is also an admissible set of indices for $X Q$.

(ii) Let $Y$ and $\bar{Y}$ be matrices of $\mathcal{M}(\underline{r}, \underline{s})$ in reduced form with the same set of indices $\underline{n}$. If there is a matrix $P \in \mathcal{G}(\underline{s})$ such that $\bar{Y}=Y P$, then $Y=\bar{Y}$.

In other words, once an admissible set of indices (i.e. linearly independent rows in the (1,1)-block) of $X \in$ $\mathcal{M}(\underline{r}, \underline{s})$ has been fixed, the reduced form, $Y$, of $X$ is unique and the free parameters of $Y$ completely parameterizes the $\left(B^{t}, A^{t}\right)$-invariant subspace $[X]$. This parameterization can be used to provide the differentiable manifold Inv $(\underline{k}, \underline{h})$ with a coordinate atlas (for the details see $[6]$ ).

The above proposition shows that all bases of the same $(C, A)$-invariant subspace have the same admissible set of indices.

Definition 2.9. .- Given a $(C, A)$-invariant subspace $\mathcal{V}$, a multiindex of $\mathcal{V}$ is any admissible set of indices for any matrix $X \in \mathcal{M}(\underline{r}, \underline{s})$ such that $\mathcal{V}=[X]$.

3. The solution of the Cover Problem. Let $\mathcal{S} \in G r_{d}\left(\mathbb{K}^{n}\right)$ and let $(A, B) \in \mathcal{M}_{n} \times \mathcal{M}_{n, m}$ be a controllable pair. As said in Section 2, we provide $\mathbb{K}^{n}$ and $\mathbb{K}^{n+m}$ with bases so that $(A, B)$ is a Brunovsky canonical matrix pair as given in [2, Lemma 4]. With regard to these bases we identify $\mathcal{S}$ with $\left[F^{*}\right], F \in \mathcal{M}_{d, n}^{*}$.

Recall that the cover problem consits of finding all matrices $X \in \mathcal{M}_{n, \delta}^{*}$ such that $[X]$ is a $\left(B^{t}, A^{t}\right)$ conditioned invariant subspace and $F X=0$. Assume that $\underline{k}=\left(k_{1}, \ldots, k_{r}\right)$ are the controllability indices of $(A, B)$ and fix a partition $\underline{h}=\left(h_{1}, \ldots, h_{s}\right)$ compatible with $\underline{k}$. Let $\underline{r}$ and $\underline{s}$ be the conjugate partitions of $\underline{k}$ and $\underline{h}$, respectively. We will restrict ourselves to find matrices $X \in \mathcal{M}(\underline{r}, \underline{s})$ such that $F X=0$. As shown in Section 2, for such matrices, $[X]$ is a $\left(B^{t}, A^{t}\right)$-conditioned invariant subspace and $\mathcal{S} \subset[X]^{\perp}$ as desired. In order to know all $\left(B^{t}, A^{t}\right)$-conditioned invariant subspace containing $\mathcal{S}$ we only have to check all possible partitions compatible with $\underline{k}$, finite in number.

Let $X \in \mathcal{M}(\underline{r}, \underline{s})$ and put $X^{j}=\left(X_{i j}\right)_{1 \leq i \leq k}, j=1, \ldots, h$. For $i=1,2, \ldots, h$ let $X_{i}$ denote the submatrix of $X$ whose $j$-th column block is the $i$-th column block of $X^{j}$. For example, for the matrix in Example 2.2 
we would have

$$
X_{1}=\left(\begin{array}{c|c|c}
X_{11}^{1} & \mathbf{0} & \mathbf{0} \\
X_{21}^{1} & \mathbf{0} & \\
0 & & \\
0 & & \\
\hline X_{11}^{2} & X_{11}^{1} & \mathbf{0} \\
0 & X_{21}^{1} & \mathbf{0} \\
0 & 0 & \\
\hline 0 & X_{11}^{2} & X_{11}^{1} \\
0 & 0 & X_{21}^{1} \\
\hline 0 & 0 & X_{11}^{2}
\end{array}\right) \quad X_{2}=\left(\begin{array}{c|c}
X_{12}^{1} & \mathbf{0} \\
X_{22}^{1} & \\
X_{32}^{1} & \\
0 & \\
\hline X_{12}^{2} & X_{12}^{1} \\
X_{22}^{2} & X_{22}^{1} \\
0 & X_{32}^{1} \\
\hline X_{12}^{3} & X_{12}^{2} \\
0 & X_{22}^{2} \\
\hline 0 & X_{12}^{3}
\end{array}\right) \quad X_{3}=\left(\begin{array}{c}
X_{13}^{1} \\
X_{23}^{1} \\
X_{33}^{1} \\
X_{43}^{1} \\
\hline X_{13}^{2} \\
X_{23}^{2} \\
X_{33}^{2} \\
\hline X_{13}^{3} \\
X_{23}^{3} \\
\hline X_{13}^{4}
\end{array}\right)
$$

Also put

$$
R_{j}^{i}=\left(\begin{array}{c}
X_{1 j}^{i} \\
X_{2 j}^{i} \\
\vdots \\
X_{k-h-i+j+1, j}^{i}
\end{array}\right), \quad j=1, \ldots, h, \quad i=1, \ldots, k-h+j
$$

and

$$
R_{j}=\left(\begin{array}{c}
R_{j}^{1} \\
R_{j}^{2} \\
\vdots \\
R_{j}^{k-h+j}
\end{array}\right), \quad j=1, \ldots, h
$$

$R_{j}$ is of size $\left(r_{h-j+1}+\cdots+r_{k}\right) \times\left(s_{h-j+1}-s_{h-j+2}\right)$ and will be said to be the condensed form of $X_{j}$, $j=1, \ldots, h$. Also the sequence of all these matrices $\left(R_{1}, \ldots, R_{h}\right)$ will be called the condensed form of $X$. Notice that by knowing the condensed form of $X$ we can easily reconstruct $X \in \mathcal{M}(\underline{r}, \underline{s})$. (The information about $\underline{r}$ and $\underline{s}$ is inside the condensed form).

In the previous example

$$
\begin{aligned}
& R_{1}^{1}=\left(\begin{array}{c}
X_{11}^{1} \\
X_{21}^{1}
\end{array}\right), \quad R_{1}^{2}=X_{11}^{2}, \quad R_{1}=\left(\begin{array}{c}
R_{1}^{1} \\
R_{1}^{2}
\end{array}\right)=\left(\begin{array}{c}
X_{11}^{1} \\
X_{21}^{1} \\
\hline X_{11}^{2}
\end{array}\right) \\
& R_{2}^{1}=\left(\begin{array}{c}
X_{12}^{1} \\
X_{22}^{1} \\
X_{32}^{1}
\end{array}\right), \quad R_{2}^{2}=\left(\begin{array}{c}
X_{12}^{2} \\
X_{22}^{2}
\end{array}\right), \quad R_{2}^{3}=X_{12}^{3}, \quad R_{2}=\left(\begin{array}{c}
X_{12}^{1} \\
X_{22}^{1} \\
X_{32}^{1} \\
\hline X_{12}^{2} \\
X_{22}^{2} \\
X_{12}^{3}
\end{array}\right)
\end{aligned}
$$

etc.

The (1,1)-block of $X, X_{11}$, will be also denoted by $R$ :

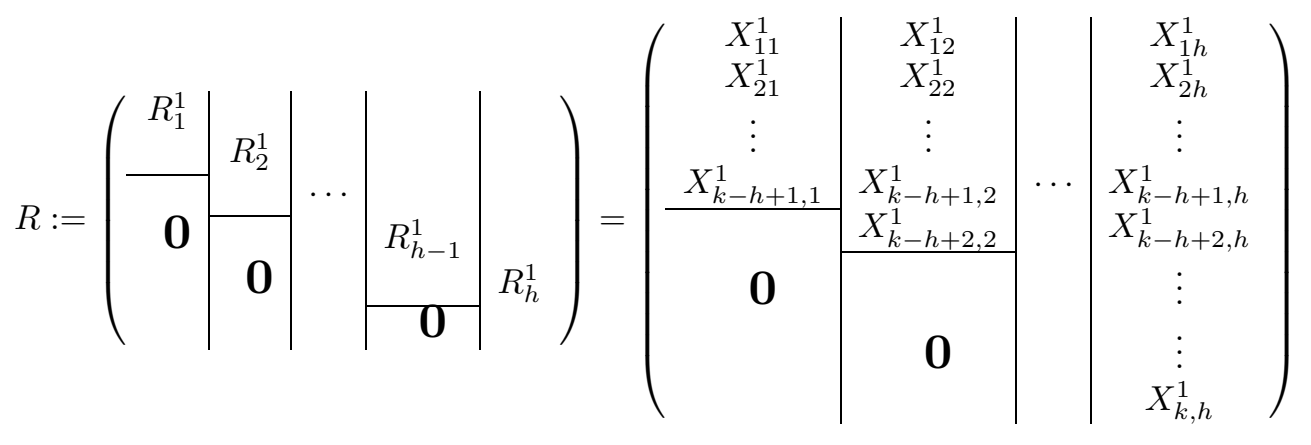


Now we split the columns of $F$ according to the sizes of the row blocks of $X$ :

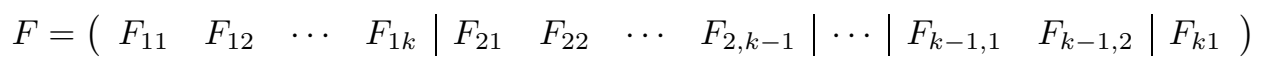

with $F_{i j}$ of size $d \times\left(r_{k-j+1}-r_{k-j+2}\right), i=1, \ldots, k, j=1, \ldots k-i+1$.

Now, $F X=0$ if and only if $F X_{j}=0$ for $j=1, \ldots, h$. In these homogeneous linear systems the unknown matrix $X_{j}$ has some repeated entries and other ones must be compulsory zero. However with the help of the matrices $R_{j}$ we can avoid these inconveniences. We introduce the following notation: For $\alpha=1, \ldots, k$ and $\beta=1, \ldots k-\alpha+1$ let

$$
H(\alpha, \beta)=\left(F_{\alpha, 1} F_{\alpha, 2} \cdots F_{\alpha, \beta}\right) \in \mathcal{M}_{d, r_{k-\beta+1}}
$$

And for $j=1, \ldots, h$ let

$$
H_{j}=\left(\begin{array}{cccc}
H(1, k-j+1) & H(2, k-j) & \cdots & H(k-j+1,1) \\
H(2, k-j+1) & H(3, k-j) & \cdots & H(k-j+2,1) \\
\vdots & \vdots & \cdots & \vdots \\
H(j, k-j+1) & H(j+1, k-j) & \cdots & H(k, 1)
\end{array}\right) .
$$

Thus $H_{j}$ is of size $j d \times\left(r_{j}+r_{j+1}+\cdots+r_{k}\right)$. Notice that this matrix is a kind of truncated block Hankel matrix. In fact

$$
\begin{aligned}
& H(2, k-j+1)=\left(H(2, k-j) F_{2, k-j+1}\right), \\
& H(3, k-j)=\left(H(3, k-j-1) F_{3, k-j}\right),
\end{aligned}
$$

and so on.

Matrices $H_{1}, \ldots, H_{h}$ only depend on the selected basis for $\mathcal{S}$ and the Brunovsky structure of $(A, B)$. We will say that $\left(H_{1}, \ldots, H_{h}\right)$ is the block-Hankel structure associated to $F$, or a block-Hankel structure of $\mathcal{S}$, with regard to $(A, B)$. (Notice that $\left.H_{1}=F\right)$.

Now it is easily computed that $F X_{j}=0$ for $j=1, \ldots, h$ if and only if

$$
H_{h-j+1} R_{j}=0, \quad j=1, \ldots, h
$$

As said above, the advantage of this expression is that all elements of $R_{j}$ can be seen as arbitrary unknowns.

Following with our example we have that

$$
F=\left(\begin{array}{llll|lll|ll|l}
F_{11} & F_{12} & F_{13} & F_{14} & F_{21} & F_{22} & F_{23} & F_{31} & F_{32} & F_{41}
\end{array}\right)
$$

and $F X=0$ if and only if

$$
\begin{aligned}
& H_{3} R_{1}=\left(\begin{array}{cc|c}
F_{11} & F_{12} & F_{21} \\
F_{21} & F_{22} & F_{31} \\
F_{31} & F_{32} & F_{41}
\end{array}\right)\left(\begin{array}{c}
X_{11}^{1} \\
X_{21}^{1} \\
\hline X_{11}^{2}
\end{array}\right)=0 \\
& H_{2} R_{2}=\left(\begin{array}{ccc|cc|c|}
F_{11} & F_{12} & F_{13} & F_{21} & F_{22} & F_{31} \\
F_{21} & F_{22} & F_{23} & F_{31} & F_{32} & F_{41}
\end{array}\right)\left(\begin{array}{c}
X_{12}^{1} \\
X_{22}^{1} \\
X_{32}^{1} \\
\hline X_{12}^{2} \\
X_{22}^{2} \\
\hline X_{12}^{3}
\end{array}\right)=0 \\
& H_{1} R_{3}=\left(\begin{array}{llllllll|ll|l|l}
F_{11} & F_{12} & F_{13} & F_{14} & F_{21} & F_{22} & F_{23} & F_{31} & F_{32} & F_{41}
\end{array}\right)\left(\begin{array}{c}
X_{13}^{1} \\
X_{23}^{1} \\
X_{33}^{1} \\
X_{43}^{1} \\
X_{13}^{2} \\
X_{23}^{2} \\
X_{33}^{2} \\
X_{13}^{3} \\
X_{23}^{3} \\
X_{13}^{4}
\end{array}\right)=0
\end{aligned}
$$


As a conclusion we have the following result whose proof is immediate

Proposition 3.1. .- With the above notation there is a matrix $X \in \mathcal{M}(\underline{r}, \underline{s})$ such that $F X=0$ if and only if there are matrices $R_{1}, \ldots, R_{h}$ such that $H_{h-j+1} R_{j}=0, j=1, \ldots, h$ and $\operatorname{rank} R=s_{1}$.

Thus the existence of solutions for the Cover Problem is reduced to the compatibility of a finite number of homogeneous linear systems with the additional restriction $\operatorname{rank} R=s_{1}$. In what follows we aim to give a more explicit condition with the help of the reduced form introduced in Definition 2.5.

First we pay attention to matrices $H_{j}$ that form a block-Hankel structure of the subspace $\mathcal{S}$. Let $t_{j}=\operatorname{rank} H_{j}, j=1, \ldots, h$. In particular $t_{1}=\operatorname{rank} H_{1}=\operatorname{rank} F=d$.

Definition 3.2. .- An increasing sequence of positive integers $1 \leq \ell_{1}^{j}<\cdots<\ell_{t_{j}}^{j} \leq r_{j}+\cdots+r_{k}$ is said to be a multiindex for $H_{j}$ if columns $\ell_{1}^{j}, \ldots, \ell_{t_{j}}^{j}$ are linearly independent.

Proposition 3.3. .- Let $F, G \in \mathcal{M}_{d, n}^{*}$ be matrices such that $\left[F^{*}\right]=\left[G^{*}\right]=\mathcal{S}$. Let $\left(H_{1}, \ldots, H_{h}\right)$ and $\left(G_{1}, \ldots, G_{h}\right)$ be two block-Hankel structures associated to $F$ and $G$ (with regard to $\left.(A, B)\right)$, respectively. Then $\operatorname{rank} H_{j}=\operatorname{rank} G_{j}$ and $\left(\ell_{1}^{j}, \ldots, \ell_{t_{j}}^{j}\right)$ is a multiindex for $H_{j}$ if and only if it is a multiindex for $G_{j}$, $j=1, \ldots, h$.

Proof.- In fact $\left[F^{*}\right]=\left[G^{*}\right]=\mathcal{S}$ if and only if there is an invertible matrix $P \in \mathcal{M}_{d}^{*}$ such that $G=P F$. Thus $G_{j}=\operatorname{Diag}(P, \ldots, P) H_{j}$ and the proposition follows

Then, the following definition makes sense. Let $F \in \mathcal{M}_{d, n}^{*}$ be such that $\left[F^{*}\right]=\mathcal{S}$ and $\left(H_{1}, \ldots, H_{h}\right)$ the block Hankel-structure associated to $F$. Let $\underline{\ell}^{j}=\left(\ell_{1}^{j}, \ldots, \ell_{t_{j}}^{j}\right)$ with $1 \leq \ell_{1}^{j}<\cdots<\ell_{t_{j}}^{j} \leq r_{j}+\cdots+r_{k}$ a multiindex for $H_{j}$. Then, we call $\underline{\ell}=\left(\underline{\ell}^{1}, \ldots, \underline{\ell}^{h}\right)$ a multiindex of $\mathcal{S}$ with respect to $(A, B)$.

Similarly we introduce a notation for the multiindices of a $(C, A)$-conditioned invariant subspace. If $\{(1 \leq$ $\left.\left.n_{i 1}<n_{i 2}<\cdots<n_{i m_{h-i+1}} \leq r_{h-i+1}\right) \mid 1 \leq i \leq h\right\}$ (recall that $\left.m_{h-i+1}=s_{h-i+1}-s_{h-i+2}\right)$ is a multiindex of a $(C, A)$-conditioned invariant subspace (Definition 2.9) then we will write $\underline{n}^{i}=\left(n_{i 1}, \ldots, n_{i m_{h-i+1}}\right)$ and $\underline{n}=\left(\underline{n}^{1}, \ldots, \underline{n}^{h}\right)$ denotes a multiindex of that subspace.

We will also use the following notation: if $\underline{i}=\left(i_{1}, \ldots, i_{r}\right)$ and $\underline{j}=\left(j_{1}, \ldots j_{s}\right)$ are sequences of positive integers such that $1 \leq i_{1}<\cdots<i_{r} \leq m$ and $1 \leq j_{1}<\cdots<j_{s} \leq n$, then $A(\underline{i}, \underline{j})$ will denote the $r \times s$ submatrix of $A \in \mathcal{M}_{m, n}$ formed with rows $i_{1}, \ldots, i_{r}$ and columns $j_{1}, \ldots, j_{s}$; and $A[\underline{i}, j]$ is the submatrix of $A$ obtained by removing rows $i_{1}, \ldots, i_{r}$ and columns $j_{1}, \ldots, j_{s}$. In particular, if $j=(1, \ldots, n)$ then $A(\underline{i}$,$) is$ the submatrix formed by rows $i_{1}, \ldots, i_{r}$ and all the columns of $A$ and $A[\underline{i}$,$] is the submatrix obtained by$ deleting from $A$ rows $i_{1}, \ldots, i_{r}$ and no columns. Similarly if $\underline{i}=(1, \ldots, m)$.

Now we can prove an explicit condition for the Cover Problem to have a solution:

ThEOREM 3.4. .- Let $\mathcal{S} \in G r_{d}\left(\mathbb{K}^{n}\right),(A, B)$ a controllable pair with $r_{1} \geq \cdots \geq r_{k}>0$ as positive Brunovsky indices and let $s_{1} \geq \cdots \geq s_{h}>0$ be a partition compatible with $\left(r_{1}, \ldots, r_{k}\right)$. Then there exists an $(A, B)$-controlled invariant subspace, $\mathcal{V} \in G r_{n-\delta}\left(\mathbb{K}^{n}\right)$ such that the restriction of $\left(B^{t}, A^{t}\right)$ to $\mathcal{V}^{\perp}$ has $\left(s_{1}, \ldots, s_{h}\right)$ as positive Brunovsky indices and $\mathcal{V} \subset \mathcal{S}$ if and only if there are multiindices $\underline{\ell}=\left(\underline{\ell}^{1}, \ldots, \underline{\ell}^{h}\right)$ and $\underline{n}=\left(\underline{n}^{1}, \ldots, \underline{n}^{h}\right)$ for $\mathcal{S}$ and $\mathcal{V}^{\perp}$, respectively, such that $\underline{\ell}^{j} \cap \underline{n}^{h-j+1}=\emptyset$ for $j=1, \ldots, h$.

Proof.- Assume first that there is an $(A, B)$-controlled invariant subspace $\mathcal{V} \in G r_{n-\delta}\left(\mathbb{K}^{n}\right)$ such that $\mathcal{S} \subset \mathcal{V}$ and let $\left[F^{*}\right]=\mathcal{S}$. For any matrix $X \in \mathcal{M}(\underline{r}, \underline{s})$ (whose columns span $\mathcal{V}^{\perp}$ ) we have that $F X=0$. By Proposition 2.6 we can assume that $X$ is in reduced form given by Definition 2.5.

Let $\underline{n}=\left(\underline{n}^{1}, \ldots, \underline{n}^{h}\right)$ be a multiindex of $\mathcal{V}^{\perp}$ and then for $X$. Since $X$ is in reduced form we have that

$$
R_{j}^{1}\left(\underline{n}^{j},\right)=I_{m_{h-j+1}}, \quad j=1, \ldots, h .
$$

Thus $\operatorname{rank} R=s_{1}$ independently of the remainder elements of $R_{i}^{1}, i=1, \ldots, h$. We only have to analyze under what conditions systems $H_{h-j+1} R_{j}=0, j=1,2, \ldots, h$, are solvable.

Let us consider system $H_{h-j+1} R_{j}=0$. As $R_{j}^{1}\left(\underline{n}^{j},\right)=I_{m_{h-j+1}}$, if $H_{h-j+1} R_{j}=0$ then

$$
H_{h-j+1}\left[, \underline{n}^{j}\right] R_{j}\left[\underline{n}^{j},\right]=-H_{h-j+1}\left(, \underline{n}^{j}\right)
$$

i.e. the columns of $H_{h-j+1}$ in $\underline{n}^{j}$ are linearly dependent on the remainder columns of $H_{h-j+1}$. Since rank $H_{h-j+1}=t_{h-j+1}$, there must be a multiindex for $H_{h-j+1}, \underline{\ell}^{h-j+1}=\left(\ell_{1}^{h-j+1}, \ldots, \ell_{t_{h-j+1}}^{h-j+1}\right)$ such that $\underline{\ell}^{h-j+1} \cap \underline{n}^{j}=\emptyset$, as desired.

Conversely, if $\underline{\ell}^{h-j+1} \cap \underline{n}^{j}=\emptyset$ for $j=1, \ldots, h$ then there are matrices $Z_{1}, \ldots, Z_{h}$ such that

$$
H_{h-j+1}\left[, \underline{n}^{j}\right] Z_{j}=-H_{h-j+1}\left(, \underline{n}^{j}\right), \quad j=1, \ldots, h .
$$


For $j=1, \ldots, h$ we construct a matrix $R_{j}$ as follows

$$
\begin{aligned}
& R_{j}\left[\underline{n}^{j},\right]=Z_{j} \\
& R_{j}\left(\underline{n}^{j},\right)=I_{m_{h-j+1}}
\end{aligned}
$$

Thus $H_{h-j+1} R_{j}=0$ and by defining $R$ as in (3.1) we have that rank $R=s_{1}$. Now the theorem follows from Proposition 3.1

It is worth noticing that in the proof of the above theorem we have not used the whole reduced form of $X$; just the fact that the rows in $\underline{n}^{j}$ are canonical vectors. Actually there are some hidden properties of the admissible multiindices of $H_{j}$ behind the block-Hankel structure of these matrices. The following one is relevant:

LEMMA 3.5. .- If for some $j=1, \ldots, h$ there is a positive integer $\alpha, 1 \leq \alpha \leq r_{j}$, such that column $\alpha$ of $H_{j}$ is a linear combination of the remainder columns of $H_{j}$ then for $i=1, \ldots, j-1$ columns $\alpha, r_{i}+$ $\alpha, \ldots, r_{i}+\cdots+r_{j-1}+\alpha$ of $H_{i}$ are also linear combinations of the remainder columns of $H_{i}$.

Proof.- Straightforward bearing in mind the block-Hankel structure of matrices $H_{j}$

A consequence of this lemma is the following necessary condition for the Cover Problem to have a solution.

THEOREM 3.6. .- Under the assumptions of Theorem 3.4 let $\left(H_{1}, \ldots, H_{h}\right)$ be any block-Hankel structure of $\mathcal{S}$ with respect to $(A, B)$. If there is an $(A, B)$-controlled invariant subspace, $\mathcal{V} \in G r_{n-\delta}\left(\mathbb{K}^{n}\right)$ such that the restriction of $\left(B^{t}, A^{t}\right)$ to $\mathcal{V}^{\perp}$ has $\left(s_{1}, \ldots, s_{h}\right)$ as positive Brunovsky indices and $\mathcal{S} \subset \mathcal{V}$ then

$$
\operatorname{rank} H_{j} \leq \sum_{i=j}^{k}\left(r_{i}-s_{i}\right), \quad j=1, \ldots, h .
$$

Proof.- We have seen in the proof of Theorem 3.4 that if $\underline{n}=\left(\underline{n}^{1}, \ldots, \underline{n}^{h}\right)$ is a multiindex for $\mathcal{V}^{\perp}$ then the columns of $H_{j}$ in $\underline{n}^{h-j+1}$ are linear combination of the remainder columns of $H_{j}$. Thus, if we agree that for a positive integer $a, a+\underline{n}^{j}=\left\{a+n_{j, 1}, a+n_{j, 2}, \ldots, a+n_{j, m_{j}}\right\}$, then, according to Lemma 3.5, the following columns of $H_{j}$ in

$$
\begin{aligned}
& \underline{n}^{h-j+1} \cup \\
& \underline{n}^{h-j} \cup\left(r_{j}+\underline{n}^{h-j}\right) \cup \\
& \underline{n}^{h-j-1} \cup\left(r_{j}+\underline{n}^{h-j-1}\right) \cup\left(r_{j}+r_{j+1}+\underline{n}^{h-j-1}\right) \cup \\
& \cdots \\
& \underline{n}^{1} \cup\left(r_{j}+\underline{n}^{1}\right) \cup \cdots\left(r_{j}+\cdots+r_{h-1}+\underline{n}^{1}\right)
\end{aligned}
$$

are linear combination of the remainder columns of $H_{j}$. But all these sets of indices are pairwise disjoint and

$$
\begin{aligned}
& \#\left(\underline{n}^{1} \cup \underline{n}^{2} \cup \cdots \cup \underline{n}^{h-j+1}\right)=s_{h}+m_{h-1}+\cdots+m_{j}=s_{j} \\
& \#\left(r_{j}+\underline{n}^{1} \cup r_{j}+\underline{n}^{2} \cup \cdots \cup r_{j}+\underline{n}^{h-j}\right)=s_{h}+m_{h-1}+\cdots+m_{j+1}=s_{j+1} \\
& \vdots \\
& \#\left(r_{1}+\cdots+r_{h-1}+\underline{n}^{1}\right)=s_{h} .
\end{aligned}
$$

As the number of columns of $H_{j}$ is $r_{j}+\cdots+r_{k}$ we conclude that

$$
\operatorname{rank} H_{j} \leq\left(r_{j}+\cdots+r_{k}\right)-\left(s_{j}+\cdots+s_{h}\right), \quad j=1, \ldots, k
$$

as desired.

Condition (3.2) is not sufficient in general because it is not only important the number of linearly independent columns of $H_{j}$ but also their positions. For example, assume that $\underline{r}=(5,3,2,1)$ and $\underline{s}=$ $(3,3,1)$ as in Example 2.7, and let $\mathcal{S}$ be the subspace of $\mathbb{R}^{11}$ generated by the matrix $F^{*}$ where $F=$ 
$\left(\begin{array}{lllllllllll}1 & 0 & 0 & 0 & 0 & 0 & 0 & 0 & 0 & 0 & 0\end{array}\right)$. Then

$$
\begin{aligned}
H_{1} & =F=\left(\begin{array}{lllll|lll|ll|l}
1 & 0 & 0 & 0 & 0 & 0 & 0 & 0 & 0 & 0 & 0
\end{array}\right) \\
H_{2} & =\left(\begin{array}{ccc|cc|c}
1 & 0 & 0 & 0 & 0 & 0 \\
0 & 0 & 0 & 0 & 0 & 0
\end{array}\right) \\
H_{3} & =\left(\begin{array}{ll|l}
1 & 0 & 0 \\
0 & 0 & 0 \\
0 & 0 & 0
\end{array}\right) .
\end{aligned}
$$

On one hand, rank $H_{1}=1 \leq r_{1}+r_{2}+r_{3}+r_{4}-s_{1}-s_{2}-s_{3}=4$, rank $H_{2}=1 \leq r_{2}+r_{3}+r_{4}-s_{2}-s_{3}=2$ and rank $H_{3}=1 \leq r_{3}+r_{4}-s_{3}=2$. Thus condition (3.2) holds. However we have seen in Example 2.7 that the only possible multiindices for all invariant subspaces are $((1),(2,3))$ and $((2),(1,3))$. (Notice that in this case $\left.\underline{n}^{3}=\emptyset\right)$. But $\ell^{1}=\ell^{2}=\ell^{3}=(1)$; and so either $\ell^{3} \cap \underline{n}^{1} \neq \emptyset$ or $\ell^{2} \cap \underline{n}^{2} \neq \emptyset$. That is to say there is not $(A, B)$-controlled invariant subspace $\mathcal{V}$ such that the restriction of $\left(B^{t}, A^{t}\right)$ to $\mathcal{V}^{\perp}$ has Brunovsky indices $\underline{s}=(3,3,1)$ and $\mathcal{S} \subset \mathcal{V}$. This does not mean that there is no $(A, B)$-invariant subspaces of codimension 7 containing $\mathcal{S}$. In fact, many other partitions $\underline{s}$ are compatible with $\underline{r}=(5,3,2,1)$. For example $\underline{s}=(4,2,1)$ is compatible with $\underline{r}$ and a possible multiindex may be $\underline{n}=((2),(3),(4,5))$. Since $\ell^{3-j+1} \cap \underline{n}^{j}=\emptyset$ for $j=1,2,3$ we conclude that there is at least one $(A, B)$-invariant subspace of codimension 7 containing $\mathcal{S}$. The orthogonal of such a subspace is generated by a matrix $Y \in \mathcal{M}(\underline{r}, \underline{s})$ in reduced form as given in Definition 2.5. Thus the orthogonal to the subspace spanned by

$$
Y\left(y_{1}, \ldots, y_{9}\right)=\left(\begin{array}{cccc|cc|c}
0 & 0 & 0 & 0 & 0 & 0 & 0 \\
1 & 0 & 0 & 0 & 0 & 0 & 0 \\
0 & 1 & 0 & 0 & 0 & 0 & 0 \\
0 & 0 & 1 & 0 & 0 & 0 & 0 \\
0 & 0 & 0 & 1 & 0 & 0 & 0 \\
\hline y_{1} & y_{2} & y_{3} & y_{4} & 0 & 0 & 0 \\
0 & 0 & 0 & 0 & 1 & 0 & 0 \\
0 & 0 & 0 & 0 & 0 & 1 & 0 \\
\hline 0 & y_{5} & y_{6} & y_{7} & y_{1} & y_{2} & 0 \\
0 & 0 & 0 & 0 & 0 & 0 & 1 \\
\hline 0 & 0 & y_{8} & y_{9} & 0 & y_{5} & y_{1}
\end{array}\right)
$$

has dimension 4 , it is $(A, B)$-invariant and contains $\mathcal{S}$. Notice that there are 9 free parameters in Y. So, there are many other different choices for matrices $Y$ in reduced form with the same properties.; i.e. each one of them spans a $\left(B^{t}, A^{t}\right)$-conditioned invariant subspace of dimension 7 with Brunovsky indices for the restriction $\underline{s}=(4,2,1)$ and whose orthogonal contains $\mathcal{S}$. We will see in a later section that the set of all $\left(B^{t}, A^{t}\right)$-conditioned invariant subspace with fixed Brunovsky indices for the restriction and whose orthogonals contain a given subspace can be provided with a differentiable structure. As we will prove, the number of free parameters in $Y$ is just the dimension of the corresponding manifold.

To finish this section is worth mentioning that, generically, condition (3.2) is also sufficient as we will show in the next section.

4. The generic case. The aim of this section is to show that, generically, that is to say for $\mathcal{S}$ belonging to an open and dense subset $\mathfrak{S}$ of $\mathrm{Gr}_{d}\left(\mathbb{K}^{n}\right)$, condition (3.2) in Theorem 3.6 is a sufficient condition for the Cover Problem to have a solution. We would like to emphasize that genericity is an interesting property from a practical point of view. In fact, it means that if $\mathcal{S} \notin \mathfrak{S}$ we can obtain by a slight modification of $\mathcal{S}$ a subspace $\mathcal{S}^{\prime} \in \mathfrak{S}$ and that if $\mathcal{S} \in \mathfrak{S}$ the small perturbations of $\mathcal{S}$ remain in $\mathfrak{S}$.

We begin by recalling the definition of genericity. Given a property $(\mathrm{P})$ concerning the elements of a topological space $T,(\mathrm{P})$ is said to be a generic property with respect to $T$ if there exists an open and dense subset $O$ of $T$ such that every element of $O$ satisfies this property. Then, we also say that the set $O$ satisfies property $(\mathrm{P})$ generically. For example, being invertible is a generic property with respect to the set of square matrices. The following property will play an important role in the achievement of our objective.

(P) We say that a matrix $A$ satisfies property (P) if $A$ has full rank, say $r$, and all $r \times r$ submatrices of $A$ are invertible.

It is clear that $(\mathrm{P})$ is a generic property with respect to $\mathcal{M}_{m, n}$. However, what we will need is that this property keeps being generic with respect to a smaller set: the set of all block-Hankel structures $\left(H_{1}, \ldots, H_{h}\right)$ associated to $F$. The following particular case is essential to this end. 
Let $\mathcal{H}_{p, q}$ be the set of $p \times q$ Hankel matrices and $\nu$ the number of perpendiculars to the main diagonal. From a topological point of view we identify $\mathcal{H}_{p, q}$ with $\mathbb{K}^{\nu}$. Let $H(x)$ be the Hankel matrix

$$
H(x)=\left(\begin{array}{ccccc}
x_{1} & x_{2} & x_{3} & \ldots & x_{q} \\
x_{2} & x_{3} & \ldots & \ldots & \ldots \\
x_{3} & \ldots & \ldots & \ldots & \ldots \\
\ldots & \ldots & \ldots & \ldots & \ldots \\
x_{p} & \ldots & \ldots & \ldots & x_{\nu}
\end{array}\right)=\left(x_{i+j-2}\right)
$$

where $x_{1}, \ldots, x_{\nu}$ are indeterminates. We will also say that $x_{1} \ldots, x_{\nu}$ are the parameters of $H(x)$ and that $H(x)$ is a parametric Hankel matrix. A particular Hankel matrix $H(a)$ is obtained by giving to the parameters the values $x_{i}=a_{i}, i=1, \ldots, \nu$. We will show that $(\mathrm{P})$ is generic with respect to $\mathcal{H}_{p, q}$. For this we need the following result

Lemma 4.1. .- Let $H(x)$ be a parametric Hankel matrix with $p \leq q$. Let $I=\left(i_{1}, \ldots, i_{p}\right)$ be a given sequence of indices, $1 \leq i_{1}<\ldots<i_{p} \leq q$, and let $\Delta_{I}(x)$ be the determinant formed with the columns in $I$. Then $\Delta_{I}(x)$ is not the zero polynomial of $\mathbb{K}[x]$.

Proof. By recurrence on the number of rows $p$. If $p=1$ the lemma is obvious. Assume now that the lemma is also true for $p-1 \geq 2$. Then, if $p$ is the number of rows of $H(x)$, we compute $\Delta_{I}(x)$ by its first column cofactor expansion:

$$
\Delta_{I}(x)=x_{i_{1}} \Delta_{1, i_{1}}(x)+\cdots+x_{i_{1}+p-1} \Delta_{p, i_{1}}(x)
$$

Notice that if we eliminate the first row of a Hankel matrix, the remaining matrix is still a Hankel matrix. Hence, by applying the induction hypothesis we conclude that $\Delta_{1, i_{1}}(x) \neq 0$. Then taking into account that the indeterminate $x_{i_{1}}$ does not appear in any of the remainder summands we obtain that $\Delta_{I}(x) \neq 0$, as desired.

Let $\mathcal{V}_{I}$ denote the set of matrices $H(a) \in \mathcal{H}_{p, q}$ such that $\Delta_{I}(a) \neq 0$. The above lemma shows that $\mathcal{V}_{I}$ is a Zariski open (and hence dense) subset of $\mathcal{H}_{p, q}$. When $I$ runs over all $i_{1}, \ldots, i_{p}$ such that $1 \leq i_{1}<\ldots<i_{p} \leq q$, $\cap_{I} \mathcal{V}_{I}$ is still open and dense. Since $H \in \mathcal{H}_{p, q}$ satisfies $(\mathrm{P})$ if and only if $H^{t}$ satisfies the same property, the next lemma follows.

LEMMA 4.2. .- $(P)$ is a generic property with respect to $\mathcal{H}_{p, q}$.

We will have to deal with matrices formed by a finite number of parametric Hankel matrices, one each other with different parameters. For convenience we introduce the following definition.

DEFINITION 4.3. Let $\mathcal{H}_{i j}\left(x_{i, j}\right)$ be a parametric Hankel matrices with different parameters (that is to say, $x_{i, j} \neq x_{k, l}$ if $\left.(i, j) \neq(k, l)\right)$. A matrix of the form

$$
\mathcal{H}(x)=\left(\begin{array}{cccc}
\mathcal{H}_{11}\left(x_{11}\right) & \mathcal{H}_{12}\left(x_{12}\right) & \cdots & H_{1 n}\left(x_{1 n}\right) \\
\mathcal{H}_{21}\left(x_{21}\right) & \mathcal{H}_{22}\left(x_{22}\right) & \cdots & H_{2 n}\left(x_{2 n}\right) \\
\vdots & \vdots & \cdots & \vdots \\
\mathcal{H}_{m 1}\left(x_{m 1}\right) & \mathcal{H}_{m 2}\left(x_{m 2}\right) & \cdots & H_{m n}\left(x_{m n}\right)
\end{array}\right)
$$

will be called an $\mathcal{H}$-matrix, For example,

$$
\left(\begin{array}{lllllllll}
x_{1} & x_{2} & x_{3} & y_{1} & y_{2} & v_{1} & v_{2} & v_{3} & v_{4} \\
x_{2} & x_{3} & x_{4} & y_{2} & y_{3} & v_{2} & v_{3} & v_{4} & v_{5} \\
& & & & & & & & \\
z_{1} & z_{2} & z_{3} & u_{1} & u_{2} & t_{1} & t_{2} & t_{3} & t_{4} \\
z_{2} & z_{3} & z_{4} & u_{2} & u_{3} & t_{2} & t_{3} & t_{4} & t_{5} \\
z_{3} & z_{4} & z_{5} & u_{3} & u_{4} & t_{3} & t_{4} & t_{5} & t_{6}
\end{array}\right)
$$

is an $\mathcal{H}$-matrix. (For simplicity we have written $x_{11}^{i}=x_{i}, x_{12}^{j}=y_{j}, \ldots$ )

LEMMA 4.4. .- $(P)$ is a generic property with respect to the set of $\mathcal{H}$-matrices. 
Proof. Let $\left(x_{i j}^{1}, \ldots, x_{i j}^{n_{i j}}\right)$ be the parameters of $\mathcal{H}_{i j}\left(x_{i j}\right)$. Since $x_{i j}^{\alpha} \neq x_{k \ell}^{\alpha}$ for $(i, j) \neq(k, \ell)$ we can obtain from $\mathcal{H}(x)$ a Hankel matrix as follows. In the first row block

$$
\left(\begin{array}{llll}
\mathcal{H}_{11}\left(x_{11}\right) & \mathcal{H}_{12}\left(x_{12}\right) & \ldots & \mathcal{H}_{1 n}\left(x_{1 n}\right)
\end{array}\right)
$$

we modify the parameters of the first column of each one of the blocks $\mathcal{H}_{12}\left(x_{12}\right), \mathcal{H}_{13}\left(x_{13}\right), \ldots$, so that the first row block becomes a Hankel matrix with different parameters. We illustrate this through the example above. Replace $y_{1}$ by $x_{4}$ and $v_{1}$ by $y_{3}$ :

$$
\left(\begin{array}{lllllllll}
x_{1} & x_{2} & x_{3} & x_{4} & y_{2} & y_{3} & v_{2} & v_{3} & v_{4} \\
x_{2} & x_{3} & x_{4} & y_{2} & y_{3} & v_{2} & v_{3} & v_{4} & v_{5}
\end{array}\right)
$$

Next we modify conveniently the parameters of the second row block

$$
\left(\begin{array}{llll}
\mathcal{H}_{21}\left(x_{21}\right) & \mathcal{H}_{22}\left(x_{22}\right) & \cdots & \mathcal{H}_{2 n}\left(x_{2 n}\right)
\end{array}\right)
$$

so that the resulting matrix formed by the two modified row blocks is a Hankel matrix with different parameters. Again, we illustrate this procedure through the previous example

$$
\left(\begin{array}{lllllllll}
x_{1} & x_{2} & x_{3} & x_{4} & y_{2} & y_{3} & v_{2} & v_{3} & v_{4} \\
x_{2} & x_{3} & x_{4} & y_{2} & y_{3} & v_{2} & v_{3} & v_{4} & v_{5} \\
& & & & & & & & \\
x_{3} & x_{4} & y_{2} & y_{3} & v_{2} & v_{3} & v_{4} & v_{5} & t_{4} \\
x_{4} & y_{2} & y_{3} & v_{2} & v_{3} & v_{4} & v_{5} & t_{4} & t_{5} \\
y_{2} & y_{3} & v_{2} & v_{3} & v_{4} & v_{5} & t_{4} & t_{5} & t_{6}
\end{array}\right)
$$

Following this way we will obtain from $\mathcal{H}(x)$ a Hankel matrix, $H(x) \in \mathcal{H}_{p, q}$. By Lemma $4.2(\mathrm{P})$ is generic with respect to $\mathcal{H}_{p, q}$, and so it is with respect to the $\mathcal{H}$-matrices.

Finally, let $\mathcal{O}_{j}$ be the set of all matrices $F \in \mathcal{M}_{d, n}$ such that if $\left(H_{1} \ldots H_{h}\right)$ is the block Hankel structure associated to $F, H_{j}$ satisfies $(\mathrm{P})$ and set $\mathcal{O}=\bigcap_{j} \mathcal{O}_{j}$.

Proposition 4.5. - $\mathcal{O}_{j}$ is an open and dense subset of $\mathcal{M}_{d, n}$.

Proof. Recall that matrices $H_{j}$, as defined in Section3, are almost block Hankel matrices. Actually we can permute the blocks in $H_{j}$ in order to obtain a matrix with the form

$$
H_{j} P=\left(\begin{array}{llll}
H_{j}^{1} & H_{j}^{2} & \cdots & H_{j}^{k_{j}}
\end{array}\right)
$$

( $P$ a permutation matrix) such that each submatrix $H_{j}^{i}$ is a block Hankel matrix for $i=1, \ldots, k_{j}$.

Following our example in Section 3 we would have

$$
\begin{array}{rlrl}
H_{1}^{1} & =\left(\begin{array}{llll}
F_{11} & F_{21} & F_{31} & F_{41}
\end{array}\right) & H_{1}^{2}=\left(\begin{array}{lll}
F_{12} & F_{22} & F_{32}
\end{array}\right) & H_{1}^{3}=\left(\begin{array}{ll}
F_{13} & F_{23}
\end{array}\right) H_{1}^{4}=F_{14} \\
H_{2}^{1}=\left(\begin{array}{lll}
F_{11} & F_{21} & F_{31} \\
F_{21} & F_{31} & F_{41}
\end{array}\right) & H_{2}^{2}=\left(\begin{array}{ll}
F_{12} & F_{22} \\
F_{22} & F_{32}
\end{array}\right) & H_{2}^{3}=\left(\begin{array}{c}
F_{13} \\
F_{23}
\end{array}\right) \\
H_{3}^{1}=\left(\begin{array}{ll}
F_{11} & F_{21} \\
F_{21} & F_{31} \\
F_{31} & F_{41}
\end{array}\right) & H_{3}^{2}=\left(\begin{array}{l}
F_{12} \\
F_{22} \\
F_{32}
\end{array}\right)
\end{array}
$$

Now, by a suitable permutation of the rows of $H_{j} P$ we can obtain an $\mathcal{H}$-matrix. The proposition follows from Lemma 4.4 taking into account that the parameters of $F$ not being in $H_{j}$ are free.

Corollary 4.6. - $\mathcal{O}$ is an open and dense subset of $\mathcal{M}_{d, n}$.

From this corollary we derive the following result which is basic to attain our objective.

Proposition 4.7. .- Let $\mathfrak{S}$ be the set of subspaces $\mathcal{S} \in G r_{d}\left(\mathbb{K}^{n}\right)$ such that $\mathcal{S}=\left[F^{*}\right]$ with $F \in \mathcal{O}$. Then $\mathfrak{S}$ is an open and dense subset of $G r_{d}\left(\mathbb{K}^{n}\right)$.

Proof. Let $F, G \in \mathcal{M}_{d, n}$ be matrices such that $\left[F^{*}\right]=\left[G^{*}\right]=\mathcal{S}$, and $\left(H_{1}, \ldots, H_{h}\right),\left(K_{1}, \ldots, K_{h}\right)$ be the block Hankel structures associated to $F$ and $G$, respectively. From Proposition 3.3 one has that $F \in \mathcal{O}$ if and 
only if $G \in \mathcal{O}$. Then the proposition follows from the above corollary and the fact that in the description of $\operatorname{Gr}_{d}\left(\mathbb{K}^{n}\right)$ as the orbit space $\mathcal{M}_{d, n}^{*} / \mathrm{Gl}\left(\mathbb{K}^{d}\right)$, the natural projection $\mathcal{M}_{d, n}^{*} \longrightarrow \mathcal{M}_{d, n}^{*} / \mathrm{Gl}\left(\mathbb{K}^{d}\right)$ is an open map.

LEMMA 4.8. .- If $\mathcal{S} \in \mathfrak{S}$ and $H_{1}, \ldots, H_{h}$ is a block Hankel structure of $\mathcal{S}$ satisfying condition (3.2) in Theorem 3.6 then

$$
\operatorname{rank} H_{j}=j d
$$

Proof. Since $\mathcal{S} \in \mathfrak{S}, H_{j}$ has full rank. Therefore,

$$
\operatorname{rank} H_{j}=\min \left\{j d, r_{j}+\cdots+r_{k}\right\}
$$

and from condition (3.2) we have

$$
\min \left\{j d, r_{j}+\cdots+r_{k}\right\} \leq \sum_{i=j}^{k}\left(r_{i}-s_{i}\right)
$$

Hence, $\operatorname{rank} H_{j}=j d$.

We are now in position to show the main result of this section.

THEOREM 4.9. .- Under the assumptions of Theorem 3.4 and with the above notation, for every $\mathcal{S} \in \mathfrak{S}$ there is an $(A, B)$-controlled invariant subspace $\mathcal{V} \in G r_{n-\delta}\left(\mathbb{K}^{n}\right)$ such that the restriction of $\left(B^{t}, A^{t}\right)$ to $\mathcal{V}^{\perp}$ has $\left(s_{1}, \ldots, s_{h}\right)$ as positive Brunovsky indices and $\mathcal{S} \subset \mathcal{V}$ if and only if

$$
0 \leq \sum_{j=h}^{k} r_{j}-s_{h}-h d
$$

Proof. (if) Condition (4.1) can be written as

$$
h d \leq \sum_{i=h}^{k}\left(r_{i}-s_{i}\right)
$$

and from this relation and bearing in mind that $r_{i} \geq s_{i}$, we conclude that for $j=1, \ldots, h$

$$
j d \leq h d \leq \sum_{i=h}^{k}\left(r_{i}-s_{i}\right) \leq \sum_{i=j}^{k}\left(r_{i}-s_{i}\right) .
$$

But by the above lemma, $j d=\operatorname{rank} H_{j}=t_{j}$. Hence,

$$
t_{j}+m_{j}=j d+s_{j}-s_{j+1} \leq r_{j}+\cdots+r_{k}, \quad j=1, \ldots, h .
$$

Since $\mathcal{S} \in \mathfrak{S}$, if $\left(H_{1}, \ldots, H_{h}\right)$ is a block-Hankel structure of $\mathcal{S}$, for $j=1, \ldots, h$ any set of columns of $H_{j}$ is linearly independent. Thus any multiindex is available to be chosen, and, in order to apply Theorem 3.4, we only have to check that $t_{j}+\# \underline{n}^{h-j+1}$ is less than or equal to the number of columns of $H_{j}$. And this is just what relation (4.3) shows.

(only if). From Theorem 3.6 we know that condition (3.2) is satisfied, so that for $j=h$ we have

$$
t_{h} \leq \sum_{j=h}^{k} r_{j}-s_{h}
$$

and from Lemma $4.8 \operatorname{rank} H_{h}=h d$. Hence, condition (4.1) is satisfied. 
REMARK 4.10. Given $\mathcal{S} \in \operatorname{Gr}_{d}\left(\mathbb{K}^{n}\right)$, the existence of an $(A, B)$-controlled invariant subspace $\mathcal{V} \in$ $\operatorname{Gr}_{n-\delta}\left(\mathbb{K}^{n}\right)$ such that the restriction of $\left(B^{t}, A^{t}\right)$ to $\mathcal{V}^{\perp}$ has $\left(s_{1}, \ldots, s_{h}\right)$ as positive Brunovsky indices and $\mathcal{S} \subset \mathcal{V}$ is equivalent to the following relation

$$
\operatorname{Gr}_{\delta}\left(\mathcal{S}^{\perp}\right) \cap \operatorname{Inv}(r, s) \neq \emptyset
$$

According to Thom Transversality Theorem a necessary condition in order the above relation to be satisfied is that

$$
\operatorname{dim} \operatorname{Gr}_{\delta}\left(\mathcal{S}^{\perp}\right)+\operatorname{dim} \operatorname{Inv}(r, s) \geq \operatorname{dim} \operatorname{Gr}_{\delta}\left(\mathbb{K}^{n}\right)
$$

That is to say, if $\eta=\operatorname{dim} \operatorname{Inv}(r, s)$ then

$$
\delta(n-d+\delta)+\eta \geq \delta(n-\delta)
$$

or

$$
\delta d \leq \eta
$$

We know that $\delta=s_{1}+\cdots+s_{h}$ and $\eta=\sum_{i=1}^{h}\left[\left(s_{i}-s_{i+1}\right) \sum_{j=1}^{k}\left(r_{i+j-1}-s_{i+j-1}\right)\right]$ (see Corollary 2.4).

Hence relation (4.5) is equivalent to

$$
d\left(\sum_{i=1}^{h} s_{i}\right) \leq \sum_{i=1}^{h}\left[\left(s_{i}-s_{i+1}\right) \sum_{j=i}^{k}\left(r_{j}-s_{j}\right)\right]
$$

But $\sum_{i=1}^{h} s_{i}=\sum_{i=1}^{h} i\left(s_{i}-s_{i+1}\right)$ so that, finally, (4.5) is equivalent to

$$
\sum_{i=1}^{h}\left[\left(s_{i}-s_{i+1}\right)\left(i d-\sum_{j=i}^{k}\left(r_{j}-s_{j}\right)\right)\right] \leq 0
$$

We then conclude that (4.1) (see (4.2)) implies (4.5). Therefore condition (4.1) in Theorem 4.9 gives a necessary condition for (4.4) to be satisfied generically. This is interesting because Thom Theorem tells us more than this. In fact, it states that the intersection in (4.4) is transversal which implies that $\operatorname{Gr}_{\delta}\left(\mathcal{S}^{\perp}\right) \cap$ Inv $(r, s)$ is a manifold and also that its dimension is given by $\eta-\delta d$. We will prove this two statements by direct methods in the next section, but not only for generic subspaces but in general.

Theorem 4.9 can be used to provide a solution, in the generic case, to the original cover problem; that is to say, without prescribing the Brunovsky indices of the restriction, $s$. This is stated in the following problem whose proof follows from the following observation. Assume that $\underline{s}^{\prime}=\left(s_{1}^{\prime}, \ldots, s_{h^{\prime}}^{\prime}\right)$ is such that $h^{\prime}<h$. Then,

$$
\sum_{j=h}^{k} r_{j}-s_{h}-h d<\sum_{j=h^{\prime}+1}^{k} r_{j}-h^{\prime} d<\sum_{j=h^{\prime}}^{k} r_{j}-s_{h^{\prime}}^{\prime}-h^{\prime} d .
$$

Corollary 4.11. .- With the notation of the above theorem, for every $\mathcal{S} \in \mathfrak{S}$ there is an $(A, B)$ controlled invariant subspace $\mathcal{V} \in G r_{n-\delta}\left(\mathbb{K}^{n}\right)$ such that $\mathcal{S} \subset \mathcal{V}$ if and only if

$$
0 \leq \sum_{j=h}^{k} r_{j}-s_{h}-h d
$$

for the unique $\underline{s}$ compatible with $\underline{r}$ having minimal $h$. 
We will give an example applying this result. First notice that the unique partition $\underline{s}$ compatible with $\underline{r}$ (i.e., $s_{i} \leq r_{i}$ for all $i$ ) having minimal $h$ can be explicitly constructed as follows (recall that $s_{1}+\cdots+s_{h}=\delta$ ):

$$
\begin{aligned}
& \text { (i) } s_{1}=\min \left\{r_{1}, \delta\right\} \\
& \text { (ii) for } j=2,3 \ldots, s_{1}+\cdots+s_{j}=\min \left\{r_{1}+\cdots+r_{j}, \delta\right\}
\end{aligned}
$$

and $h=\min \left\{j: r_{1}+\cdots r_{j} \geq \delta\right\}$.

EXAMPLE 4.12. Let $\underline{r}=(3,3,2)$ and $d=3$. We are going to determine when the cover problem has generically solution for the possible values of $\delta$. Since $n=8$, we have $1 \leq \delta \leq 4$. We examine the inequality of (4.6) for the partitions $\underline{s}$ compatible with $\underline{r}$ having minimal $h$, that is to say, for $\delta=1, \underline{s}=(1)$, for $\delta=2$, $\underline{s}=(2)$, for $\delta=3, \underline{s}=(3)$ and for $\delta=4, \underline{s}=(3,1)$. We conclude that for $\delta=1,2,3$ the cover problem has generically solution and for $\delta=4$, it does not.

5. The differentiable structure of the solutions. Along this section we are given a subspace $\mathcal{S} \in G r_{d}\left(\mathbb{K}^{n}\right)$ for what there is at least one $\mathcal{V} \in \operatorname{Inv}(\underline{r}, \underline{s})$ such that $\mathcal{S} \subset \mathcal{V}^{\perp}$. A necessary and sufficient condition for such a property to hold was given in Theorem 3.4. Our aim in this section is to show that for subspace $\mathcal{S}$ the set $\operatorname{Inv}(\underline{r}, \underline{s}, \mathcal{S})=\left\{\mathcal{V} \in \operatorname{Inv}(\underline{r}, \underline{s}) \mid \mathcal{S} \subset \mathcal{V}^{\perp}\right\}$ can be provided with a differentiable structure, to compute its dimension and to find an atlas of coordinate charts. This will allow us to parameterize the solutions of the Cover Problem.

To begin with let $F \in \mathcal{M}_{d, n}^{*}$ be a matrix such that $\mathcal{S}=\left[F^{*}\right]$ and let $X \in \mathcal{M}(\underline{r}, \underline{s})$ be such that $\mathcal{V}=[X]$ with $\mathcal{V} \in \operatorname{Inv}(\underline{r}, \underline{s}, \mathcal{S})$. Then $F X=0$. Let

$$
\mathcal{N}(\underline{r}, \underline{s}, \mathcal{S}):=\{X \in \mathcal{M}(\underline{r}, \underline{s}) \mid F X=0\} .
$$

Notice that if $F, G \in \mathcal{M}_{d, n}^{*}$ such that $\mathcal{S}=\left[F^{*}\right]=\left[G^{*}\right]$ then $\{X \in \mathcal{M}(\underline{r}, \underline{s}) \mid F X=0\}=\{X \in \mathcal{M}(\underline{r}, \underline{s}) \mid G X=$ $0\}$. For notational simplicity we will write $\mathcal{N}:=\mathcal{N}(\underline{r}, \underline{s}, \mathcal{S})$ and $\mathcal{M}:=\mathcal{M}(\underline{r}, \underline{s})$. Let $\mathcal{G}:=\mathcal{G}(\underline{s})$ be the group defined in Definition 2.1. By Theorem 2.3 this group acts freely on $\mathcal{M}$ on the right by matrix multiplication and so does it on $\mathcal{N}$. In addition we can identify $\operatorname{Inv}(\underline{r}, \underline{s}, \mathcal{S})$ with $\mathcal{N} / \mathcal{G}$ by means of the bijection

$$
\begin{array}{ccc}
\mathcal{N} / \mathcal{G} & \longrightarrow & \operatorname{Inv}(\underline{r}, \underline{s}, \mathcal{S}) \\
\{X P \mid X \in \mathcal{N}, P \in \mathcal{G}(\underline{s})\} & \rightsquigarrow & {[X]}
\end{array}
$$

Through this bijection a differentiable structure on $\operatorname{Inv}(\underline{r}, \underline{s}, \mathcal{S})$ can be defined:

THEOREM 5.1. .- (i) The orbit space $\mathcal{N} / \mathcal{G}$ has a differentiable stucture such that the natural projection $\pi: \mathcal{N} \rightarrow \mathcal{N} / \mathcal{G}$ is a submersion.

(ii) $\operatorname{dim}(\mathcal{N} / \mathcal{G})=\operatorname{dim} \mathcal{N}-\operatorname{dim} \mathcal{G}$.

Proof.- (i) According to [7, Th 2.9.10] we have to prove:

(a) $\Gamma=\left\{\left(X, X^{\prime}\right) \in \mathcal{N} \times \mathcal{N} \mid X^{\prime}=X P, P \in \mathcal{G}\right\}$ is closed in $\mathcal{N} \times \mathcal{N}$, and

(b) the map $\gamma: \mathcal{N} \times \mathcal{G} \rightarrow \Gamma$ defined by $\gamma(X, P)=(X, X P)$ is a homeomorphism.

In order to prove (a) notice that if $\Gamma_{1}=\left\{\left(X, X^{\prime}\right) \in \mathcal{M} \times \mathcal{M} \mid X^{\prime}=X P, P \in \mathcal{G}\right\}$ then $\Gamma=\Gamma_{1} \cap(\mathcal{N} \times \mathcal{N})$. As $\Gamma_{1}$ is closed in $\mathcal{M} \times \mathcal{M}$ (see [5]), $\Gamma$ is closed in $\mathcal{N} \times \mathcal{N}$.

On the other hand, $\gamma$ is clearly bijective due to the free action of $\mathcal{G}$ on $\mathcal{N}$, and it is continue because it is the restriction of the homeomorphism $\gamma_{1}: \mathcal{M} \times \mathcal{G} \rightarrow \Gamma_{1}$ defined by $\gamma_{1}(X, P)=(X, X P)$ (see again [5]). Its inverse is also continue for the same reason.

(ii) The dimension formula follows from (i) as in [5].

Now we compute the dimension of $\mathcal{N} / \mathcal{G}$. On one hand (see [6])

$$
\operatorname{dim} \mathcal{G}=\sum_{i=1}^{k} \sum_{j=1}^{h}\left(s_{i}-s_{i+1}\right) s_{i+j-1}
$$

And on the other hand $X \in \mathcal{N}$ if and only if $X \in \mathcal{M}$ and $F X=0$. As shown in the previous section this is equivalent to

$$
H_{h-j+1} R_{j}=0, \quad j=1, \ldots, h,
$$

where $\left(R_{1}, \ldots, R_{h}\right)$ is the condensed form of $X$ and $\left(H_{1}, \ldots, H_{h}\right)$ is a block-Hankel structure of $\mathcal{S}$ with respect to $(A, B)$. This is equivalent, in turns, to

$$
H_{h-j+1} R_{j}(, i)=0, \quad j=1, \ldots, h, i=1, \ldots, s_{h-j+1}-s_{h-j+2},
$$


where, as in Section $3, R_{j}(, i)$ is the ith column of $R_{j}$. The set of solutions of this linear homogeneous system is a vector space of dimension $\left(r_{h-j+1}+\cdots+r_{k}\right)-\operatorname{rank} H_{h-j+1}$; i.e. the solutions depend on $\left(r_{h-j+1}+\cdots+r_{k}\right)-\operatorname{rank} H_{h-j+1}$ parameters. Thus the solutions of $H_{h-j+1} R_{j}=0$ depend on $\left(s_{h-j+1}-\right.$ $\left.s_{h-j+2}\right)\left(\sum_{i=h-j+1}^{k} r_{i}-\operatorname{rank} H_{h-j+1}\right)$ parameters. Since we have $h$ of these systems,

$$
\operatorname{dim} \mathcal{N}=\sum_{j=1}^{h}\left(s_{j}-s_{j+1}\right)\left(\sum_{i=j}^{k} r_{i}-\operatorname{rank} H_{j}\right)=\sum_{j=1}^{h}\left(s_{j}-s_{j+1}\right)\left(\sum_{i=1}^{k} r_{i+j-1}-\operatorname{rank} H_{j}\right) .
$$

Hence we have proved

Proposition 5.2. .- $\operatorname{dim} \mathcal{N} / \mathcal{G}=\sum_{j=1}^{h}\left(s_{j}-s_{j+1}\right)\left(\sum_{i=1}^{k}\left(r_{i+j-1}-s_{i+j-1}\right)-\operatorname{rank} H_{j}\right)$.

Recall that (see [6])

$$
\operatorname{dim} \mathcal{M} / \mathcal{G}=\sum_{j=1}^{h} \sum_{i=1}^{k}\left(s_{j}-s_{j+1}\right)\left(r_{i+j-1}-s_{i+j-1}\right)
$$

Hence

$$
\operatorname{dim} \mathcal{N} / \mathcal{G}=\operatorname{dim} \mathcal{M} / \mathcal{G}-\sum_{j=1}^{h}\left(s_{j}-s_{j+1}\right) \operatorname{rank} H_{j}
$$

Notice that, in the generic case, by Lemma $4.8 \operatorname{rank} H_{j}=j d$, and so $\sum_{j=1}^{h}\left(s_{j}-s_{j+1}\right) \operatorname{rank} H_{j}=d \sum_{j=1}^{h} j\left(s_{j}-\right.$ $\left.s_{j+1}\right)=d \sum_{j=1}^{h} s_{j}=d \delta$. Since $\eta=\operatorname{dim} \operatorname{Inv}(\underline{r}, \underline{s})=\operatorname{dim} \mathcal{M} / \mathcal{G}$, we recover the result in Remark 4.10.

Since $\mathcal{N} / \mathcal{G} \subset \mathcal{M} / \mathcal{G}$ and the topology of $\mathcal{N} / \mathcal{G}$ is the induced one by that of $\mathcal{M} / \mathcal{G}$ ( $\mathcal{N}$ is a submanifold of $\mathcal{M}$ and the projection $\mathcal{M} \rightarrow \mathcal{M} / \mathcal{G}$ is an open map), we have the following

PROPOSITION 5.3. $\mathcal{N} / \mathcal{G}$ is a regular submanifold of $\mathcal{M} / \mathcal{G}$.

We are going now to parameterize $\mathcal{N} / \mathcal{G}$ by means of a coordinate atlas.

First we recall an elementary fact from linear algebra. If the homogeneous linear system $A x=0$ has a solution, $\left\{a_{i_{1}}, \ldots, a_{i_{r}}\right\}$ is a basis of the column span of $A$ and $\left\{j_{1}, \ldots, j_{n-r}\right\}=\{1, \ldots, n\} \backslash\left\{i_{1}, \ldots, i_{r}\right\}$ then

$$
a_{j_{k}}=\sum_{t=1}^{r} \alpha_{k t} a_{i_{t}}, \quad k=1, \ldots, n-r
$$

if and only if

$$
x_{i_{t}}=-\sum_{k=1}^{n-r} \alpha_{t k} x_{j_{k}}, \quad t=1, \ldots, r
$$

Consider now a matrix $X \in \mathcal{M}(\underline{r}, \underline{s})$ in reduced form (Definition 2.5) such that $F X=0$, and for $j=1, \ldots, h$ let $\left(R_{1}, \ldots, R_{h}\right)$ be the condensed form of $X$. Let $\underline{\ell}=\left(\underline{\ell}^{1}, \ldots, \underline{\ell}^{k}\right)$ and $\underline{n}=\left(\underline{n}^{1}, \ldots, \underline{n}^{h}\right)$ be compatible multiindices of $\mathcal{S}$ and $X$, respectively; i.e.

$$
\underline{\ell}^{j} \cap \underline{n}^{h-j+1}=\emptyset, \quad j=1, \ldots, h .
$$

If $\underline{\ell}^{j}=\left(\underline{\ell}_{1}^{j}, \ldots, \underline{\ell}_{t_{j}}^{j}\right), t_{j}=\operatorname{rank} H_{j}$, then $\ell_{1}^{j}, \ldots, \ell_{t_{j}}^{j}$ are linearly independent columns of $H_{j},\left(H_{1}, \ldots, H_{h}\right)$ being a block-Hankel structure of $\mathcal{S}$ with respect to $(A, B)$.

Since $X$ is in reduced form, the rows of $R_{h-j+1}$ in $\underline{n}^{h-j+1}$ are canonical vectors; i.e. $R_{h-j+1}\left(\underline{n}^{h-j+1},\right)=$ $I_{m_{j}}$. And the rows of $R_{h-j+1}$ in the set of indices $\underline{p}^{h-j+1}=\underline{n}^{1} \cup \cdots \cup \underline{n}^{h-j} \cup\left(r_{j}+\underline{n}^{1}\right) \cup \cdots \cup\left(r_{j}+\underline{n}^{h-j}\right) \cup\left(r_{j+1}+\right.$ $\left.r_{j}+\underline{n}^{1}\right) \cup \cdots \cup\left(r_{j+1}+r_{j}+\underline{n}^{h-j-1}\right) \cup \cdots \cup\left(r_{k}+\cdots+r_{j}+\underline{n}^{1}\right)$ are zero rows; i.e. $R_{h-j+1}\left(\underline{p}^{h-j+1},\right)=0$. 
(Compare with the indices in the proof of Theorem 3.6). But $F X=0$ is equivalent to $H_{j} R_{h-j+1}=0$, $j=1, \ldots, h$. So, if $s^{j}=\# \underline{p}^{h-j+1}=2 s_{j+1}+s_{j+2}+\ldots+s_{h}, r^{j}=r_{j}+\cdots+r_{k}$ and

$$
\left\{g_{1}^{j}, \ldots, g_{r^{j}-t_{j}-s^{j}}^{j}\right\}=\left\{1, \ldots, r^{j}\right\} \backslash\left(\underline{\ell}^{j} \cup \underline{p}^{h-j+1}\right)
$$

then columns $H_{j}\left(, g_{i}^{j}\right)$ are linear combinations of the columns $\ell_{1}^{j}, \ldots, \ell_{t_{j}}^{j}$ of $H_{j}$. We can write

$$
H_{j}\left(, g_{i}^{j}\right)=\sum_{q=1}^{t_{j}} \alpha_{i q} H_{j}\left(, \ell_{q}^{j}\right), \quad i=1, \ldots, r^{j}-t_{j}-s^{j},
$$

and so

$$
R_{h-j+1}\left(\ell_{i}^{j},\right)=-\sum_{q=1}^{r^{j}-t_{j}-s^{j}} \alpha_{q i} R_{h-j+1}\left(g_{q}^{j},\right), \quad i=1, \ldots, t_{j} .
$$

This proves the following

TheOrem 5.4. .- For any matrix $X \in \mathcal{N}(\underline{r}, \underline{s}, \mathcal{S})$ there are multiindices $\underline{\ell}=\left(\underline{\ell}^{1}, \ldots, \underline{\ell}^{h}\right)$ of $\mathcal{S}$ and $\underline{n}=\left(\underline{n}^{1}, \ldots, \underline{n}^{h}\right)$ of $X$ such that

(i) $\ell^{j} \cap \underline{n}^{h-j+1}=\emptyset, \quad j=1, \ldots, h$.

(ii) $Y=X P$ is in reduced form, as give by Definition 2.5 , for some $P \in \mathcal{G}(\underline{s})$.

(iii) if $\left(H_{1}, \ldots, H_{h}\right)$ is a block-Hankel structure of $\mathcal{S},\left(R_{1}, \ldots, R_{h}\right)$ the condensed form of $Y,\left\{g_{1}^{j}, \ldots\right.$, $\left.g_{r^{j}-t_{j}-s^{j}}^{j}\right\}=\left\{1, \ldots, r^{j}\right\} \backslash\left(\underline{\ell}^{j} \cup \underline{p}^{h-j+1}\right)$ for $j=1, \ldots, h$ and $H_{j}\left(, g_{i}^{j}\right)$ is given by (5.1) then matrix $R_{h-j+1}\left(\ell_{i}^{j},\right)$ is given by (5.2).

DEFINITION 5.5. .- With the notation of the above theorem we will say that $Y$ is a reduced form of $X \in \mathcal{N}(\underline{r}, \underline{s}, \mathcal{S})$ with respect to $\underline{n}$ and $\underline{\ell}$.

Let us compute now the number of free parameters in a reduced form of $X \in \mathcal{N}(\underline{r}, \underline{s}, \mathcal{S})$. We will show that this is the dimension of $\mathcal{N} / \mathcal{G}$. In fact, the number of free parameters of any reduced form is the same as the number of free parameters in its condensed form. And this is the number of free parameters in the condensed form of the reduced form of $X \in \mathcal{M}(\underline{r}, \underline{s})$ minus the number of elements in $R_{h-j+1}\left(\ell_{i}^{j},\right)$, $j=1, \ldots, h, i=1, \ldots, t_{j}$. Bearing in mind that the number of free parameters in any reduced form $Y \in \mathcal{M}(\underline{r}, \underline{s})$ is $\operatorname{dim} \mathcal{M} / \mathcal{G}$ (see [6]), we conclude that the number of free parameters in $Y \in \mathcal{N}(\underline{r}, \underline{s}, \mathcal{S})$ is (recall that the number of columns of $R_{h-j+1}$ is $s_{j}-s_{j+1}$ )

$$
\operatorname{dim} \mathcal{M} / \mathcal{G}-\sum_{j=1}^{h} t_{j}\left(s_{j}-s_{j+1}\right)=\operatorname{dim} \mathcal{N} / \mathcal{G}
$$

Definition 5.6. .- A multiindex $\underline{n}=\left(\underline{n}^{1}, \ldots, \underline{n}^{h}\right)$ for $X \in \mathcal{M}(\underline{r}, \underline{s})$ will be said an admissible set of indices (or a multiindex) for $X \in \overline{\mathcal{N}}(\underline{r}, \underline{s}, \overline{\mathcal{S}})$ if there is a multiindex $\underline{\ell}=\left(\underline{\ell}^{1}, \ldots, \underline{\ell}^{h}\right)$ of $\mathcal{S}$ such that $\underline{\ell}^{j} \cap \underline{n}^{h-j+1}=\emptyset, j=1, \ldots, h$.

The following lemmas follow immediately from Lemmas 3 and 4 of [6] (see Proposition 2.8.

Lemma 5.7. .- Let $X \in \mathcal{N}$ and $Q \in \mathcal{G}$. If $\underline{n}$ is an admissible set of indices for $X$ it is also an admissible set of indices for $X Q$.

LEMMA 5.8. .- Let $Y$ and $\tilde{Y}$ be matrices of $\mathcal{N}(\underline{r}, \underline{s}, \mathcal{S})$ in reduced form with the same multiindex $\underline{n}$. If there is a matrix $P \in \mathcal{G}$ such that $\tilde{Y}=Y P$ then $P$ is the identity matrix.

In order to define the coordinate charts of $\mathcal{N} / \mathcal{G}$ we start as in [6]. Let $\Lambda$ be the set of multiindices $\underline{n}=\left(\underline{n}^{1}, \ldots, \underline{n}^{h}\right)$ for which there is a multiindex, $\underline{\ell}=\left(\underline{\ell}^{1}, \ldots, \underline{\ell}^{k}\right)$, of $\mathcal{S}$ with respect to $(A, B)$ such that $\underline{\ell}^{j} \cap \underline{n}^{h-j+1}=\emptyset, j=1, \ldots, h$; i.e. compatible with $\underline{n}$. For $\underline{n} \in \Lambda$ let $\mathcal{U}_{\underline{n}}$ denote the set of matrices $X \in \mathcal{N}(\underline{r}, \underline{s}, \mathcal{S})$ with $\underline{n}$ as a set of admissible indices. Since all matrices in $\mathcal{N}(\underline{r}, \underline{s}, \mathcal{S})$ close enough to $X$ have $\underline{n}$ as a set of admissible indices (because these are just linear independent rows), we have that $\left\{\mathcal{U}_{\underline{n}} \mid \underline{n} \in \bar{\Lambda}\right\}$ is an open covering of $\mathcal{N}(\underline{r}, \underline{s}, \mathcal{S})$. If $\pi: \mathcal{N} \rightarrow \mathcal{N} / \mathcal{G}$ is the natural projection then $\pi$ is open and $\left\{\tilde{\mathcal{U}}_{\underline{n}}=\pi\left(\mathcal{U}_{\underline{n}}\right) \mid \underline{n} \in \Lambda\right\}$ is an open covering of $\mathcal{N} / \mathcal{G}$.

We must notice that for a multiindex $\underline{n} \in \Lambda$, there may be several multiindices $\underline{\ell}$ compatible with it. To define the coordinate charts we aim to find a differentiable onto mapping $\phi_{\underline{n}}: \mathcal{U}_{\underline{n}} \rightarrow \mathbb{K}^{N}, N=\operatorname{dim} \mathcal{N} / \mathcal{G}$, for each $\underline{n} \in \Lambda$. We proceed as follows. If $\underline{n} \in \Lambda$ admits several compatible multiindices $\underline{\ell}=\left(\underline{\ell}^{1}, \ldots, \underline{\ell}^{h}\right)$ 
we take the one such that for $i=1, \ldots, h, \underline{\ell}^{i}$ is the smallest in the lexicographical order. In other words, as the elements of $\underline{\ell}^{i}$ are linearly independent columns of $H_{i}$, we are taking its first $t_{i}$ linearly independent columns compatible with $\underline{n}$. Then for $X \in \mathcal{U}_{\underline{n}}$ we define $\phi_{\underline{n}}(X)$ as the point of $\mathbb{K}^{N}$ defined by the free parameters of the reduced form of $X$ corresponding to $\underline{n}$ and $\underline{\ell}, Y$, in a certain order. For example, if $\left(R_{1}, \ldots, R_{h}\right)$ is the condensed form of $Y$, then the free parameters of $Y$ are in the rows $\left\{f_{1}^{j}, \ldots, f_{r^{j}-t_{j}-s^{\prime j}}^{j}\right\}=$ $\left\{1, \ldots, r^{j}\right\} \backslash\left(\underline{\ell}^{j} \cup \underline{p}^{h-j+1} \cup \underline{n}^{h-j+1}\right)\left(s^{\prime j}=s_{j}+s_{j+1}+\cdots+s_{h}\right)$ of $R_{h-j+1}, j=1, \ldots, h$. Thus we may define

$$
\phi_{\underline{n}}(X)=\left(R_{1}\left(f_{1}^{1},\right) R_{1}\left(f_{2}^{1},\right) \cdots R_{1}\left(f_{r^{j}-t_{1}-s^{\prime 1}}^{1},\right) R_{2}\left(f_{1}^{2},\right) \cdots R_{2}\left(f_{r^{j}-t_{2}-s^{\prime 2}}^{2},\right) \cdots R_{h}\left(f_{r^{j}-t_{h}-s^{\prime h}}^{h},\right)\right) .
$$

In this way $\phi_{\underline{n}}$ is well defined and onto. The differentiability follows from the fact that $Y_{\underline{n}}$ is obtained form $X$ by means of rational operations. This mapping induces the mapping $\theta_{\underline{n}}: \tilde{\mathcal{U}}_{\underline{n}} \rightarrow \mathbb{K}^{N}$.

THEOREM 5.9. .- With the above notation $\theta_{\underline{n}}$ is a diffeomorphism and $\left\{\tilde{\mathcal{U}}_{\underline{n}} ; \underline{n} \in \Lambda\right\}$ is a coordinate atlas of $\mathcal{N} / \mathcal{G}$

Proof. As in [6].

EXAMPLE 5.10. .- Consider a slight modification of the example at the end of the Section 3: $\underline{r}=$ $(5,3,2,1), \underline{s}=(3,3,1)$ and $\mathcal{S}=\left[F^{*}\right]$ where $F=\left(\begin{array}{ccccccccccc}1 & 0 & 0 & 0 & 0 & 2 & 0 & 0 & 0 & 0 & 0\end{array}\right)$. Then

$$
\begin{aligned}
& H_{1}=F=\left(\begin{array}{ccccc|ccc|cc|c}
1 & 0 & 0 & 0 & 0 & 2 & 0 & 0 & 0 & 0 & 0
\end{array}\right) \\
& H_{2}=\left(\begin{array}{lll|ll|l}
1 & 0 & 0 & 2 & 0 & 0 \\
2 & 0 & 0 & 0 & 0 & 0
\end{array}\right) \\
& H_{3}=\left(\begin{array}{ll|l}
1 & 0 & 2 \\
2 & 0 & 0 \\
0 & 0 & 0
\end{array}\right) \text {. }
\end{aligned}
$$

An admissible multiindex in $\mathcal{M}(\underline{r}, \underline{s})$ is $\underline{n}=((2),(3),(4,5))$. But now this multiindex is compatible with two multiindices $\underline{\ell}: \underline{\ell}_{1}=((1),(1,4),(1,3))$ and $\underline{\ell}_{2}=((6),(1,4),(1,3))$. The one to be chosen to construct the coordinate chart is $\underline{\ell}_{1}$. The reduced form corresponding to $\underline{n}$ and $\underline{\ell}_{1}$ is

$$
Y=\left(\begin{array}{cccc|cc|c}
0 & 0 & -2 a_{2} & -2 a_{3} & 0 & 0 & 0 \\
1 & 0 & 0 & 0 & 0 & 0 & 0 \\
0 & 1 & 0 & 0 & 0 & 0 & 0 \\
0 & 0 & 1 & 0 & 0 & 0 & 0 \\
0 & 0 & 0 & 1 & 0 & 0 & 0 \\
\hline 0 & 0 & a_{2} & a_{3} & 0 & 0 & 0 \\
0 & 0 & 0 & 0 & 1 & 0 & 0 \\
0 & 0 & 0 & 0 & 0 & 1 & 0 \\
\hline 0 & a_{1} & a_{4} & a_{5} & 0 & 0 & 0 \\
0 & 0 & 0 & 0 & 0 & 0 & 1 \\
\hline 0 & 0 & a_{6} & a_{7} & a_{4} & a_{5} & 0
\end{array}\right),
$$

and so $\phi_{\underline{n}}\left(\tilde{\mathcal{U}}_{\underline{n}}\right)=\left\{\left(a_{1}, a_{2}, a_{3}, a_{4}, a_{5}, a_{6}, a_{7}\right) \in \mathbb{R}^{7}\right\}=\mathbb{R}^{7}$.

Our last results is about the connection of the manifold $\mathcal{N} / \mathcal{G}$

TheOREM 5.11. .- If $\mathbb{K}=\mathbb{C}$, the manifold $\mathcal{N} / \mathcal{G}$ is connected.

Proof.- It is sufficient to prove that $\mathcal{N}$ is connected.

Assume, on the contrary, that there are open sets $U_{1}, U_{2} \in \mathcal{N}$ such that $U_{1} \cup U_{2}=\mathcal{N}$ and $U_{1} \cap U_{2}=\emptyset$. Let $X_{1} \in U_{1}$ and $X_{2} \in U_{2}$ and let $\underline{n}$ be an admissible multiindex for $X_{1}$. For a given matrix $X \in \mathcal{N}$, let $M_{\underline{n}}(X)$ denote the submatrix of $X$ formed by the rows in $\underline{n}$ and the first $s_{1}$ columns. As $\underline{n}$ is a multiindex admissible for $X_{1}$ we have that $\operatorname{det} M_{n}\left(X_{1}\right) \neq 0$.

For $z \in \mathbb{C}$ define $p(z)=\operatorname{det} M_{\underline{n}}\left(X_{1}+z\left(X_{2}-X_{1}\right)\right)$. Since $p(0)=\operatorname{det} M_{\underline{n}}\left(X_{1}\right) \neq 0, p(z)$ is not the zero polynomial, and so $p(z)$ has finitely many zeros. This allows us to define a trajectory $z(t):[0,1] \rightarrow \mathbb{C}$ such that $z(0)=0, z(1)=1$ and $p(z(t)) \neq 0$ for all $t \in[0,1)$. In other words, if $X(t)=X_{1}+z(t)\left(X_{2}-X_{1}\right)$ then $\operatorname{det} M_{n}(X(t)) \neq 0$ for all $t \in[0,1)$. Notice that for $t \in[0,1), X(t) \in \mathcal{N}=U_{1} \cup U_{2}$. Furthermore, since $X_{1} \in U_{1},\{X(t) \mid t \in[0,1)\}$ is connected (because it is the image of a connected set by a continuous map) and $U_{1} \cap U_{2}=\emptyset$ we conclude that $\{X(t) \mid t \in[0,1)\} \subset U_{1}$.

Now as $X_{2} \in U_{2}$ and $U_{2}$ is open there is a neibourhood of $X_{2}$ contained in $U_{2}$. But $X_{2}$ is the limit of a sequence of matrices in $\{X(t) \mid t \in[0,1)\}$. This means that in that neibourhood of $X_{2}$ there are matrices of this set. Thus $U_{1} \cap U_{2} \neq \emptyset$. A contradiction. 
6. Conclusions. In this paper the solutions of the cover problem has been studied by taking a geometric approach. The main tool is the stratification of the set of $(C, A)$-conditioned invariant subspaces according to the Brunovsky indices of the restriction, each strata being a differentiable manifold. The structure of the matrices whose columns form a basis of such $(C, A)$-invariant subspaces (with respect to the dual Brunovsky basis of $\mathbb{K}^{n}$ and $\mathbb{K}^{n+m}$ ) has been used to produce a necessary and sufficient condition for the cover problem to have a solution in each strata. This condition has been used to provide the set of solutions with a differentiable structure in such a way that this set is a regular submanifold of the previous one. Then, an atlas of coordinate charts for the set of solution has been given, obtaining a parameterization of all solutions of the cover problem on each strata. The generic case has been also considered. In this case a simple and very easily computable condition for the cover problem to have a solution has been shown to hold.

\section{REFERENCES}

[1] A. C. Antoulas, New results on the Algebraic Theory of Linear Systems;: The solution of the Cover Problems, Linear Algebra Appl., 50, 1-43 (1983).

[2] I. Baragaña, I. Zaballa, Block Similarity invariants of restrictions to (A, B)-invariant subspaces. Lienar Algebra Appl., 220, 31-62 (1995)

[3] G. Basile, G. Marro, Controlled and Conditioned Invariants in Linear System Theory Prentice Hall, Englewood Clifs, New Jersey, (1991).

4] P. Brunovsky, Classification of Linear Controllable Systems. Kybernetika. (Praga), 3 (6), 173-188 (1970).

[5] J. Ferrer, F. Puerta, X. Puerta, Differentiable structure of the set of the controllable $(A, B)^{t}$-invariant subspace. Linear Algebra Appl., 275-276, 161-177, (1998).

[6] F. Puerta, X. Puerta, I. Zaballa, A coordinate atlas of the manifold of observable conditioned invariant subspaces. Int. J. Control, 74 (12), 1226-1238, (2001).

[7] V. S. Varadarajan, Lie Groups, Lie Algebras and Their Representations. Springer-Verlag, New York 1984.

[8] W. M. Wonham, Linear Multivariable Control: A Geometrical Approach. Springer-Verlag, New York, 1984. 